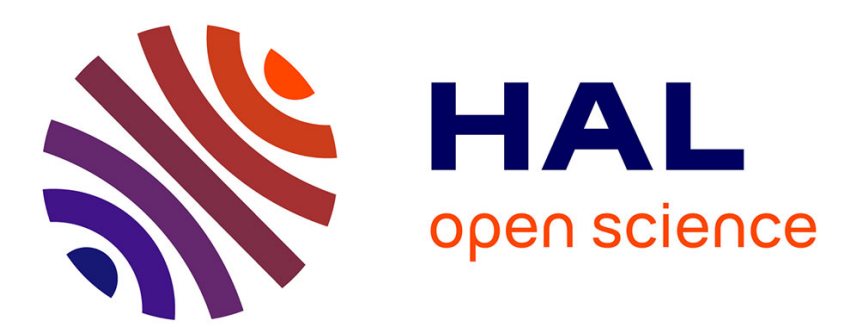

\title{
A new third-order shear deformation theory with non-linearities in shear for static and dynamic analysis of laminated doubly curved shells
}

\author{
Marco Amabili
}

\section{- To cite this version:}

Marco Amabili. A new third-order shear deformation theory with non-linearities in shear for static and dynamic analysis of laminated doubly curved shells. Composite Structures, 2015, 128, pp.260-273. 10.1016/j.compstruct.2015.03.052 . hal-01298744

HAL Id: hal-01298744

https://hal.science/hal-01298744

Submitted on 6 Apr 2016

HAL is a multi-disciplinary open access archive for the deposit and dissemination of scientific research documents, whether they are published or not. The documents may come from teaching and research institutions in France or abroad, or from public or private research centers.
L'archive ouverte pluridisciplinaire HAL, est destinée au dépôt et à la diffusion de documents scientifiques de niveau recherche, publiés ou non, émanant des établissements d'enseignement et de recherche français ou étrangers, des laboratoires publics ou privés. 


\title{
A new third-order shear deformation theory with non-linearities in shear for static and dynamic analysis of laminated doubly curved shells
}

\author{
Marco Amabili \\ Department of Mechanical Engineering, McGill University, 817 Sherbrooke Street West, Montreal H3A 0C3, Canada E-mail address: marco.amabili@mcgill.ca
}

\begin{abstract}
A geometrically nonlinear theory is developed for shells of generic shape allowing for third order shear deformation and rotary inertia by using five parameters: in plane and transverse displacements and the two rotations of the normal; geometric imperfections are also taken into account. The novelty is that geo metrically nonlinear strain displacement relationships are derived retaining full nonlinear terms in all the five parameters. These relationships are presented in curvilinear coordinates, ready to be implemented in computer codes. Higher order terms in the transverse coordinate are retained in the derivation so that the theory is suitable also for thick laminated shells. The theory is applied to laminated composite circular cylindrical shells complete around the circumference and simply supported at both ends. Initially static finite deformation and buckling due to lateral pressure is studied. Finally, large amplitude forced vibra tions under radial harmonic excitation are investigated by using the new theory and results are compared to another third order shear deformation theory that neglects nonlinear terms in rotations of the normal.
\end{abstract}

Shell theory, Shear deformation, Nonlinear static analysis, Nonlinear vibrations, Shells, Laminated

\section{Introduction}

Classical shell theories neglect shear deformation and rotary inertia and overestimate natural frequencies of moderately thick or laminated anisotropic shells and plates (e.g. [1 3 ]). They use three parameters, i.e. the displacements in the three directions of points on the shell middle surface, to describe the shell deforma tion. The geometrically nonlinear version of classical shell theories can be dividend in those that keep only von Kármán type nonlinear terms (i.e. those involving the normal displacement only) and those that retain nonlinear terms also for the two in plane displacements. The theories that retain only von Kármán type nonlinear terms are known for being accurate only for small displacements. A simplified version of them uses the Airy potential stress function to reduce the problem to a single parameter, which is the normal displacement, by neglecting in plane inertia. This theory is known as the Donnell nonlinear shallow shell theory and can be applied to complete circular shells only for circum ferential wavenumbers larger than 4 or 5 , so that in plane inertia becomes negligible.

* Address: Department of Mechanical Engineering, McGill University, Macdonald Engineering Building, 817 Sherbrooke Street West, Montreal, PQ H3A 0C3, Canada. Tel.: +1 514398 3068; fax: +1 5143987365 .
In order to overcome the limitation of classical shell theories, shear deformation theories have been introduced [1,3,4]. They use five independent parameters in order to describe the shell deformation (the three displacements of points of the middle surface and the two rotations of the transverse normal to the middle surface with respect to the in plane curvilinear coordinate lines). A classification in first order (uniform shear through the thickness) and higher order shear deformation theories can be introduced. Versions of shear deformation theories suitable for finite deformation can retain only von Kármán type nonlinear terms [5,6] or also in plane nonlinear terms [7]. The theory developed by Amabili and Reddy [7] belongs to the class of the equivalent single layer (ESL) theories. It must be observed that another class of theories, the layer wise model (LWM) has also been developed, but not retaining geometrically nonlinear terms, see e.g. Carrera [8]. At present, no shear deformation theory that retains geometrically nonlinear terms in rotations of the normal and in the shear strains is available. A review of recent studies on nonlinear vibrations of shells is given by Alijani and Amabili [9].

Shear deformation theories, which are obtained under the hypothesis of zero transverse normal stress, are accurate unless the shell presents significant thickness deformation. Shells made of soft materials (e.g. rubbers or biological materials) can present very large deformations, still in the linear material regime or in 
hyperelastic regime, associated to large thickness reduction (e.g. arteries under internal pressure or inflated rubber balloons). The effect of transverse normal stress in multilayered plates and shells and their natural vibrations has been studied by Carrera $[8,10]$ by using a model that considers continuity of interlaminar transverse and shear stresses and zig zag form of the displacement distribution in the shell thickness.

An accurate linear shell theory that takes into account thickness stretching has been developed by Carrera et al. [11] and Ferreira et al. [12]. In particular, in reference [12] natural frequencies of laminated spherical and cylindrical panels are investigated by using this theory. Also Parisch [13], Sansour [14] and Sansour et al. [15] have been developed independently shell theories that introduce quadratic assumption of the transverse shell displacement over the shell thickness for finite element imple mentation. In particular, Sansour [14] and Sansour et al. [15] use 7 independent parameters to describe the shell deformation.

A first order shear deformation shell theory with seven parame ters and exact nonlinear deformations, under the framework of the Lagrangian description, has been derived in tensor notation by Arciniega and Reddy [16,17] for laminated and functionally graded shells. This theory also uses quadratic expansion of the shell transverse displacement over the shell thickness. A similar theory has been used by Payette and Reddy [18] in conjunction with an high order spectral/hp type quadrilateral finite element technol ogy. Amabili [19] introduced a first order thickness stretching the ory with higher order shear deformation that uses 6 independent parameters. Amabili [20] introduced a geometrically nonlinear shell theory allowing for third order thickness stretching, higher order shear deformation and rotary inertia by using eight parame ters. It seems to be the first study using 8 parameters to describe the shell nonlinear deformation. In $[19,20]$ nonlinear terms in the middle plane displacements are retained but those on rotations and thickness deformation are neglected. Full nonlinear terms for displacements, rotations and thickness deformation were intro duced by Alijani and Amabili [21] for plates and Amabili [22] for shells of generic shape.

In the present study, a geometrically nonlinear theory is devel oped for shells of generic shape allowing for third order shear deformation and rotary inertia by using five parameters: in plane and transverse displacements and the two rotations of the normal; geometric imperfections are also taken into account. The novelty is that geometrically nonlinear strain displacement relationships are derived retaining full nonlinear terms in all the five parameters. These relationships are presented in curvilinear coordinates, ready to be implemented in computer codes. Higher order terms in the transverse coordinate are retained in the derivation so that the theory is suitable also for thick laminated shells. The theory is applied to laminated composite circular cylindrical shells complete around the circumference and simply supported at both ends. Initially static finite deformation and buckling due to lateral pressure is studied. Finally, large amplitude forced vibrations under radial harmonic excitation are investigated by using the new theory and results are compared to another third order shear deformation theory that neglects nonlinear terms in rotations of the normal.

\section{Geometrically nonlinear third-order shear deformation theory}

A laminated composite shell of arbitrary shape, made of a finite number of orthotropic layers, oriented arbitrarily with respect to the shell principal curvilinear coordinates $\left(\alpha_{1}, \alpha_{2}\right)$ is considered. The theory is also valid for shells made of isotropic or functionally graded materials. The displacements of an arbitrary point of coordinates $\left(\alpha_{1}, \alpha_{2}\right)$ taken on the middle surface of the shell are denoted by $u, v$ and $w$, in the $\alpha_{1}, \alpha_{2}$ and $z$ directions, respectively.
The normal displacement $w$ is taken positive outward from the center of the smallest radius of curvature, as shown in Fig. 1. In the figure $R_{1}$ and $R_{2}$ (functions of the coordinates $\alpha_{1}$ and $\alpha_{2}$ ) are the principal radii of curvature in $\alpha_{1}$ and $\alpha_{2}$ directions, respectively. Initial geometric imperfections of the shell associated with zero initial tension are denoted by displacement $w_{0}$ in $z$ direction, also taken positive outward and measured from the ideal middle surface. Imperfections other than in normal directions are not considered and an initial stress free state is assumed.

The thickness $h$ of the shell is assumed to be smaller of the principal radii of curvature of the shell, but not very small, so that moderately thick shells can be considered with accuracy. Fig. 2 shows the displacements $\left(u_{1}, u_{2}, u_{3}\right)$ in $\alpha_{1}, \alpha_{2}$ and $z$ directions, respectively, of a generic point of the shell at distance $z$ from the middle surface. These displacements $\left(u_{1}, u_{2}, u_{3}\right)$ are related to the middle surface displacements and rotations of the transverse normal by

$u_{1} \quad\left[1+\left(z / R_{1}\right)\right] u+z \phi_{1}+z^{2} \psi_{1}+z^{3} \gamma_{1}$,

$u_{2} \quad\left[1+\left(z / R_{2}\right)\right] v+z \phi_{2}+z^{2} \psi_{2}+z^{3} \gamma_{2}$

$u_{3} \quad w+w_{0}$,

where $\phi_{1}$ and $\phi_{2}$ are the rotations of the transverse normal at $z=0$ (i.e. referred to the middle surface) due to bending (but not shear deformation) about the $\alpha_{2}$ and $\alpha_{1}$ axes, respectively. Then $\psi_{1}, \psi_{2}$, $\gamma_{1}$ and $\gamma_{2}$, are functions to be determined in terms of $u, v, w, \phi_{1}$ and $\phi_{2}$, which are the five parameters describing the shell deforma tion. In Eq. (1) all the displacements have been expanded up to the 3rd order in $z$. Eqs. (1a,b) give a parabolic distribution of shear strains through the thickness, while Eq. (1c) gives a uniform trans verse normal strain displacement through the shell thickness.

The expressions of the Green's strains $\varepsilon_{11}, \varepsilon_{22}$ and $\gamma_{12}$ in the curvilinear coordinate system, are obtained from the theory of surfaces and the shell deformation as in $\left[\begin{array}{lll}1,7,22 & 24]\end{array}\right.$

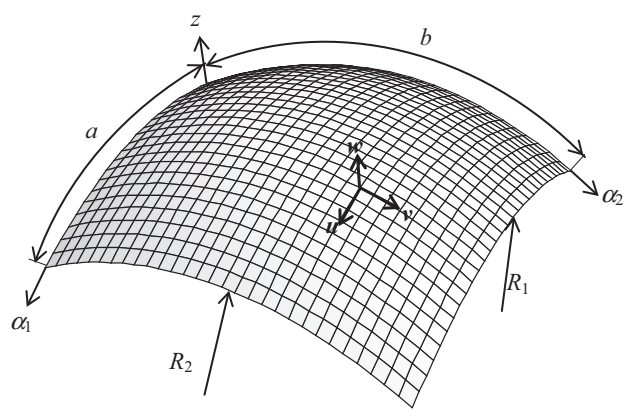

Fig. 1. Doubly curved shell.

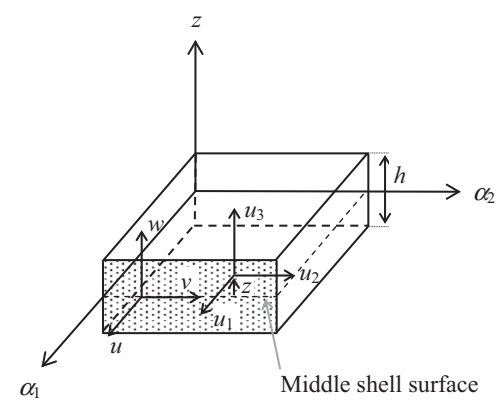

Fig. 2. Displacements of a point on the middle surface of the shell and of a generic point at distance $z$ from the middle surface. 


$$
\begin{array}{ll}
\varepsilon_{11} & \varepsilon_{1}+\frac{1}{2}\left(\varepsilon_{1}^{2}+\omega_{1}^{2}+\Theta^{2}\right), \\
\varepsilon_{22} & \varepsilon_{2}+\frac{1}{2}\left(\omega_{2}^{2}+\varepsilon_{2}^{2}+\Psi^{2}\right), \\
\gamma_{12} & \omega_{1}+\omega_{2}+\left(\varepsilon_{1} \omega_{2}+\varepsilon_{2} \omega_{1}+\Theta \Psi\right),
\end{array}
$$

where

$$
\begin{aligned}
& \varepsilon_{1} \frac{1}{1+\left(z / R_{1}\right)}\left(\frac{1}{A_{1}} \frac{\partial u_{1}}{\partial \alpha_{1}}+\frac{1}{A_{1} A_{2}} \frac{\partial A_{1}}{\partial \alpha_{2}} u_{2}+\frac{u_{3}}{R_{1}}\right), \\
& \varepsilon_{2} \frac{1}{1+\left(z / R_{2}\right)}\left(\frac{1}{A_{2}} \frac{\partial u_{2}}{\partial \alpha_{2}}+\frac{1}{A_{1} A_{2}} \frac{\partial A_{2}}{\partial \alpha_{1}} u_{1}+\frac{u_{3}}{R_{2}}\right) \text {, }
\end{aligned}
$$

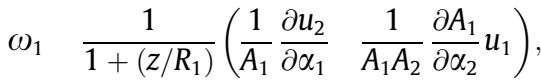

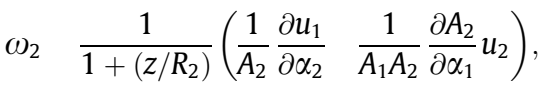

$$
\begin{aligned}
& \Theta \frac{1}{1+\left(z / R_{1}\right)}\left(\frac{1}{A_{1}} \frac{\partial u_{3}}{\partial \alpha_{1}}+\frac{u_{1}}{R_{1}}\right) \text {, } \\
& \Psi \frac{1}{1+\left(z / R_{2}\right)}\left(\frac{1}{A_{2}} \frac{\partial u_{3}}{\partial \alpha_{2}}+\frac{u_{2}}{R_{2}}\right) .
\end{aligned}
$$

In Eq. (3) $A_{1}$ and $A_{2}$ are the Lamé parameters. The corresponding strain displacement equations, keeping terms up to $z^{3}$ and using approximations

$\frac{1}{1+(z / R)} \simeq 1 \quad \frac{z}{R}+\frac{z^{2}}{R^{2}}$

can be written in the following expressions

$$
\begin{array}{ll}
\varepsilon_{11} & \varepsilon_{1,0}+z\left(k_{1}^{(0)}+z k_{1}^{(1)}+z^{2} k_{1}^{(2)}\right), \\
\varepsilon_{22} & \varepsilon_{2,0}+z\left(k_{2}^{(0)}+z k_{2}^{(1)}+z^{2} k_{2}^{(2)}\right), \\
\gamma_{12} & \gamma_{12,0}+z\left(k_{12}^{(0)}+z k_{12}^{(1)}+z^{2} k_{12}^{(2)}\right),
\end{array}
$$

where the terms in Eq. (5) have to be determined. In particular, $\varepsilon_{1,0}, \varepsilon_{2,0}$ and $\gamma_{12,0}$ represent the middle surface strains, $k_{1}^{(0)}, k_{1}^{(1)}, k_{1}^{(2)}, k_{2}^{(0)}, k_{2}^{(1)}$ and $k_{2}^{(2)}$ the change in curvature in direction 1 and 2 , respectively, and $k_{12}^{(0)}, k_{12}^{(1)}, k_{12}^{(2)}$ the change in torsion.

The shear Green's strains for three dimensional elasticity are $[1,23]:$

$$
\begin{aligned}
& \gamma_{13} \frac{\partial u_{1}}{\partial z}+\frac{1}{1+\left(z / R_{1}\right)}\left[\left(\begin{array}{ll}
\frac{1}{A_{1}} \frac{\partial u_{3}}{\partial \alpha_{1}} & \frac{u_{1}}{R_{1}}
\end{array}\right)\right. \\
& +\left(\frac{1}{A_{1}} \frac{\partial u_{1}}{\partial \alpha_{1}}+\frac{1}{A_{1} A_{2}} \frac{\partial A_{1}}{\partial \alpha_{2}} u_{2}+\frac{u_{3}}{R_{1}}\right) \frac{\partial u_{1}}{\partial z} \\
& \left.+\left(\frac{1}{A_{1}} \frac{\partial u_{2}}{\partial \alpha_{1}} \quad \frac{1}{A_{1} A_{2}} \frac{\partial A_{1}}{\partial \alpha_{2}} u_{1}\right) \frac{\partial u_{2}}{\partial z}+\left(\begin{array}{ll}
\frac{1}{A_{1}} \frac{\partial u_{3}}{\partial \alpha_{1}} & \frac{u_{1}}{R_{1}}
\end{array}\right) \frac{\partial u_{3}}{\partial z}\right],
\end{aligned}
$$

$$
\begin{aligned}
& \gamma_{23} \frac{\partial u_{2}}{\partial z}+\frac{1}{1+\left(z / R_{2}\right)}\left[\left(\begin{array}{ll}
\frac{1}{A_{2}} \frac{\partial u_{3}}{\partial \alpha_{2}} & \frac{u_{2}}{R_{2}}
\end{array}\right)\right. \\
& +\left(\frac{1}{A_{2}} \frac{\partial u_{2}}{\partial \alpha_{2}}+\frac{1}{A_{1} A_{2}} \frac{\partial A_{2}}{\partial \alpha_{1}} u_{1}+\frac{u_{3}}{R_{2}}\right) \frac{\partial u_{2}}{\partial z}
\end{aligned}
$$

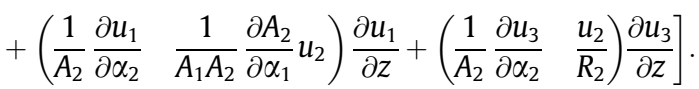

Eqs. (6a,b) are nonlinear in the displacements $u_{1}, u_{2}, u_{3}$. For thick shells, shear deformation should be retained in order to obtain accurate results. The expressions for the transverse shear strains are obtained by substituting Eqs. (1a c) into (6a,b) and neglecting nonlinear terms in the five parameters that are multiplied by $z$ and powers of $z$. The vanishing of the shear stresses $\tau_{13}$ and $\tau_{23}$ at the top and the bottom surfaces of the shell requires can be written in the following expressions

$\left.\gamma_{13}\right|_{z \pm h / 2} \quad 0,\left.\quad \gamma_{23}\right|_{z \pm h / 2} \quad 0$, since $\tau_{13} \quad G_{13} \gamma_{13}$ and $\tau_{23} \quad G_{23} \gamma_{23}$, where $G_{13}$ and $G_{23}$ are the shear moduli in 13 and 23 directions, respectively. Eqs. (7a,b) are used in order to determine $\psi_{1}, \psi_{2}, \gamma_{1}$ and $\gamma_{2}$ as functions of the eight vari ables that describe the shell deformation. In particular, it is possible to reorganize the terms in the shear strains as a polynomial in $z$

$\gamma_{13} \quad a_{1}+b_{1} z+c_{1} z^{2}+d_{1} z^{3}, \gamma_{23} a_{2}+b_{2} z+c_{2} z^{2}+d_{2} z^{3}$.

Nonlinear terms in Eqs. (8a,b) appear just in $a_{1}$ and $a_{2}$. Eqs. (7a,b) rewritten for the expressions $(8 \mathrm{a}, \mathrm{b})$ give

$\left\{\begin{array}{ll}4 b_{1}+h^{2} d_{1} & 0, \\ 4 a_{1}+h^{2} c_{1} & 0,\end{array} \quad \begin{cases}4 b_{2}+h^{2} d_{2} & 0, \\ 4 a_{2}+h^{2} c_{2} & 0 .\end{cases}\right.$

The following two expressions are obtained from Eq. (9a)

$$
\begin{aligned}
& \left.\phi_{1} \frac{4}{R_{1}}+\frac{h^{2}}{R_{1}^{3}}\right)+\psi_{1}\left(8+\frac{h^{2}}{R_{1}^{2}}\right) \quad \frac{h^{2}}{R_{1}} \gamma_{1} \frac{4}{R_{1}} \frac{\partial\left(w+w_{0}\right)}{A_{1} \partial \alpha_{1}} 0 \\
& 3 h^{3} \gamma_{1} \frac{h^{2}}{R_{1}} \psi_{1}+\phi_{1}\left(4+\frac{h^{2}}{R_{1}^{2}}\right)+\frac{\partial\left(w+w_{0}\right)}{A_{1} \partial \alpha_{1}}\left(4+\frac{h^{2}}{R_{1}^{2}}\right) \\
& +4\left(\frac{\partial u}{A_{1} \partial \alpha_{1}}+\frac{1}{A_{1} A_{2}} \frac{\partial A_{1}}{\partial \alpha_{2}} v+\frac{w+w_{0}}{R_{1}}\right)\left(\frac{u}{R_{1}}+\phi_{1}\right) \\
& +4\left(\frac{\partial v}{A_{1} \partial \alpha_{1}} \frac{1}{A_{1} A_{2}} \frac{\partial A_{1}}{\partial \alpha_{2}} u\right)\left(\frac{v}{R_{2}}+\phi_{2}\right) \quad 0 .
\end{aligned}
$$

The nonlinear terms in Eq. (10b) can be neglected for consis tency since it has been assumed that all the nonlinear terms multi plied by $z$ and its powers can be neglected so nonlinear terms in $\psi_{1}$, $\psi_{2}, \gamma_{1}$ and $\gamma_{2}$ should be also neglected, see Eqs. (1a,b).

Neglecting terms of the order $h /\left(R_{1}\right)^{2}$ with respect to unity since terms in $z^{4}$ are neglected in the expressions of $u_{1}$ and $u_{2}$, Eqs. $(10 a, b)$ give

$$
\begin{aligned}
& \psi_{1} \frac{1}{3 R_{1}} \phi_{1}+\frac{1}{3 R_{1}} \frac{\partial\left(w+w_{0}\right)}{A_{1} \partial \alpha_{1}}, \\
& \gamma_{1} \frac{4}{3 h^{2}}\left(\frac{\partial\left(w+w_{0}\right)}{A_{1} \partial \alpha_{1}}+\phi_{1}\right) .
\end{aligned}
$$

Similarly, from Eq. (9b), the following expressions are obtained:

$$
\begin{aligned}
& \psi_{2} \frac{1}{3 R_{2}} \phi_{2}+\frac{1}{3 R_{2}} \frac{\partial\left(w+w_{0}\right)}{A_{2} \partial \alpha_{2}}, \\
& \gamma_{2} \frac{4}{3 h^{2}}\left(\frac{\partial\left(w+w_{0}\right)}{A_{2} \partial \alpha_{2}}+\phi_{2}\right) .
\end{aligned}
$$

By substituting Eqs. (1a c) into (6a,b) and using Eqs. (4), (11) and (12), the following strain displacement relationships are obtained for the shear strains keeping terms up to $z^{3}$ :

$$
\begin{array}{ll}
\gamma_{13} & \gamma_{13,0}+z\left(k_{13}^{(0)}+z k_{13}^{(1)}+z^{2} k_{13}^{(2)}\right), \\
\gamma_{23} & \gamma_{23,0}+z\left(k_{23}^{(0)}+z k_{23}^{(1)}+z^{2} k_{23}^{(2)}\right),
\end{array}
$$

where

$$
\begin{aligned}
& \gamma_{13,0} \quad \phi_{1}+\frac{\partial w}{A_{1} \partial \alpha_{1}}+\left(\frac{w+w_{0}}{R_{1}}+\frac{\partial u}{A_{1} \partial \alpha_{1}}\right)\left(\frac{u}{R_{1}}+\phi_{1}\right) \\
& +\frac{\partial v}{A_{1} \partial \alpha_{1}}\left(\frac{v}{R_{2}}+\phi_{2}\right)+\frac{u v}{A_{1}} \frac{\partial A_{1}}{A_{2} \partial \alpha_{2}}\left(\begin{array}{ll}
\frac{1}{R_{1}} & \frac{1}{R_{2}}
\end{array}\right) \\
& +\frac{\partial A_{1}}{A_{1} A_{2} \partial \alpha_{2}}\left(\phi_{1} v \quad \phi_{2} u\right) \text {, } \\
& \gamma_{23,0} \quad \phi_{2}+\frac{\partial w}{A_{2} \partial \alpha_{2}}+\left(\frac{w+w_{0}}{R_{2}}+\frac{\partial v}{A_{2} \partial \alpha_{2}}\right)\left(\frac{v}{R_{2}}+\phi_{2}\right) \\
& +\frac{\partial u}{A_{2} \partial \alpha_{2}}\left(\frac{u}{R_{1}}+\phi_{1}\right)+\frac{u v}{A_{2}} \frac{\partial A_{2}}{A_{1} \partial \alpha_{1}}\left(\begin{array}{ll}
\frac{1}{R_{2}} & \frac{1}{R_{1}}
\end{array}\right) \\
& +\frac{\partial A_{2}}{A_{1} A_{2} \partial \alpha_{1}}\left(\phi_{2} u \quad \phi_{1} v\right) \text {, }
\end{aligned}
$$




$$
\begin{aligned}
& k_{13}^{(0)} \quad \frac{1}{3 R_{1}}\left(\phi_{1}+\frac{\partial w}{A_{1} \partial \alpha_{1}}\right), \\
& k_{23}^{(0)} \quad \frac{1}{3 R_{2}}\left(\phi_{2}+\frac{\partial w}{A_{2} \partial \alpha_{2}}\right) \text {, } \\
& k_{13}^{(1)} \quad \frac{4}{h^{2}}\left(\phi_{1}+\frac{\partial w}{A_{1} \partial \alpha_{1}}\right), \\
& k_{23}^{(1)} \quad \frac{4}{h^{2}}\left(\phi_{2}+\frac{\partial w}{A_{2} \partial \alpha_{2}}\right), \\
& k_{13}^{(2)} \frac{4}{3 h^{2} R_{1}}\left(\phi_{1}+\frac{\partial w}{A_{1} \partial \alpha_{1}}\right) \quad \frac{u}{R_{1}^{4}}, \\
& k_{23}^{(2)} \frac{4}{3 h^{2} R_{2}}\left(\phi_{2}+\frac{\partial w}{A_{2} \partial \alpha_{2}}\right) \quad \frac{v}{R_{2}^{4}} \text {. }
\end{aligned}
$$

Eqs. (13) (17) show that the strain and stress distribution through the thickness is of cubic order. In particular, Eqs. (14a,b) retains nonlinear terms that have been neglected in previous study by Amabili and Reddy [7].

The expressions of the middle surface strains and changes in curvature and torsion in Eq. (5) are obtained substituting Eqs. (1a c) into (2) and making use of Eqs. (3) (5), (11) and (12)

$$
\begin{aligned}
\varepsilon_{1,0} & \frac{1}{A_{1}} \frac{\partial u}{\partial \alpha_{1}}+\frac{1}{A_{1} A_{2}} \frac{\partial A_{1}}{\partial \alpha_{2}} v+\frac{w}{R_{1}}+\frac{1}{2}\left[\left(\frac{\partial u}{A_{1} \partial \alpha_{1}}+\frac{1}{A_{1} A_{2}} \frac{\partial A_{1}}{\partial \alpha_{2}} v+\frac{w}{R_{1}}\right)^{2}\right. \\
+ & \left.\left(\frac{\partial v}{A_{1} \partial \alpha_{1}} \frac{1}{A_{1} A_{2}} \frac{\partial A_{1}}{\partial \alpha_{2}} u\right)^{2}+\left(\frac{\partial w}{A_{1} \partial \alpha_{1}} \frac{u}{R_{1}}\right)^{2}\right] \\
+ & \frac{w_{0}}{R_{1}}\left(\frac{\partial u}{A_{1} \partial \alpha_{1}}+\frac{1}{A_{1} A_{2}} \frac{\partial A_{1}}{\partial \alpha_{2}} v+\frac{w}{R_{1}}\right)+\frac{\partial w_{0}}{A_{1} \partial \alpha_{1}}\left(\begin{array}{ll}
\frac{\partial w}{A_{1} \partial \alpha_{1}} & \frac{u}{R_{1}}
\end{array}\right),
\end{aligned}
$$

$\varepsilon_{2,0} \quad \frac{1}{A_{2}} \frac{\partial v}{\partial \alpha_{2}}+\frac{1}{A_{1} A_{2}} \frac{\partial A_{2}}{\partial \alpha_{1}} u+\frac{w}{R_{2}}+\frac{1}{2}\left[\left(\frac{1}{A_{2}} \frac{\partial u}{\partial \alpha_{2}} \frac{1}{A_{1} A_{2}} \frac{\partial A_{2}}{\partial \alpha_{1}} v\right)^{2}\right.$

$$
\left.+\left(\frac{1}{A_{2}} \frac{\partial v}{\partial \alpha_{2}}+\frac{1}{A_{1} A_{2}} \frac{\partial A_{2}}{\partial \alpha_{1}} u+\frac{w}{R_{2}}\right)^{2}+\left(\frac{1}{A_{2}} \frac{\partial w}{\partial \alpha_{2}} \frac{v}{R_{2}}\right)^{2}\right]
$$$$
+\frac{w_{0}}{R_{2}}\left(\frac{1}{A_{2}} \frac{\partial v}{\partial \alpha_{2}}+\frac{1}{A_{1} A_{2}} \frac{\partial A_{2}}{\partial \alpha_{1}} u+\frac{w}{R_{2}}\right)+\frac{\partial w_{0}}{A_{2} \partial \alpha_{2}}\left(\frac{\partial w}{A_{2} \partial \alpha_{2}} \frac{v}{R_{2}}\right),
$$

$\gamma_{12,0} \quad \frac{1}{A_{1}} \frac{\partial v}{\partial \alpha_{1}}+\frac{1}{A_{2}} \frac{\partial u}{\partial \alpha_{2}} \quad \frac{1}{A_{1} A_{2}} \frac{\partial A_{1}}{\partial \alpha_{2}} u \quad \frac{1}{A_{1} A_{2}} \frac{\partial A_{2}}{\partial \alpha_{1}} v$

$$
\begin{aligned}
& +\left(\frac{1}{A_{1}} \frac{\partial u}{\partial \alpha_{1}}+\frac{1}{A_{1} A_{2}} \frac{\partial A_{1}}{\partial \alpha_{2}} v+\frac{w}{R_{1}}\right)
\end{aligned}
$$

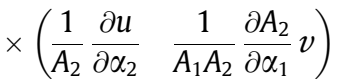

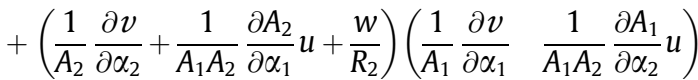

$$
\begin{aligned}
& +\left(\begin{array}{lll}
\frac{1}{A_{1}} \frac{\partial w}{\partial \alpha_{1}} & \frac{u}{R_{1}}
\end{array}\right)\left(\begin{array}{ll}
\frac{1}{A_{2}} \frac{\partial w}{\partial \alpha_{2}} & \frac{v}{R_{2}}
\end{array}\right) \\
& +\frac{w_{0}}{R_{1}}\left(\frac{1}{A_{2}} \frac{\partial u}{\partial \alpha_{2}} \quad \frac{1}{A_{1} A_{2}} \frac{\partial A_{2}}{\partial \alpha_{1}} v\right)
\end{aligned}
$$

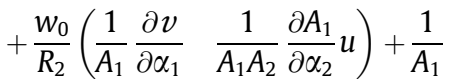

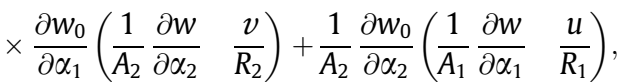

$k_{1}^{(0)} \quad \frac{\partial \phi_{1}}{A_{1} \partial \alpha_{1}} \quad \frac{w}{R_{1}^{2}}+\frac{v}{A_{1} A_{2}} \frac{\partial A_{1}}{\partial \alpha_{2}}\left(\frac{1}{R_{1}}+\frac{1}{R_{2}}\right)+\frac{\phi_{2}}{A_{1} A_{2}} \frac{\partial A_{1}}{\partial \alpha_{2}}$,

$$
\begin{aligned}
& \left.k_{1}^{(1)} \quad \frac{w}{R_{1}^{3}} \quad \frac{1}{3 R_{1} A_{1}} \quad 2 \frac{\partial \phi_{1}}{\partial \alpha_{1}}+\frac{\partial A_{1}}{A_{1}^{2} \partial \alpha_{1}} \frac{\partial w}{\partial \alpha_{1}} \quad \frac{\partial^{2} w}{A_{1} \partial \alpha_{1}^{2}}\right) \\
& \left.\frac{\partial A_{1}}{A_{1} A_{2} \partial \alpha_{2}}\left[\begin{array}{ll}
\phi_{2}\left(\frac{1}{R_{1}}\right. & \frac{1}{3 R_{2}}
\end{array}\right)+\frac{v}{R_{1}}\left(\begin{array}{ll}
\frac{1}{R_{2}} & \frac{1}{R_{1}}
\end{array}\right) \quad \frac{\partial w}{3 R_{2} A_{2} \partial \alpha_{2}}\right] \text {, }
\end{aligned}
$$

$$
\begin{aligned}
k_{1}^{(2)}= & \left.\frac{4}{3 h^{2}} \frac{\partial \phi_{1}}{A_{1} \partial \alpha_{1}}+\frac{\partial^{2} w}{A_{1}^{2} \partial \alpha_{1}^{2}}+\frac{\phi_{2}}{A_{1} A_{2}} \frac{\partial A_{1}}{\partial \alpha_{2}} \quad \frac{\partial w}{A_{1}^{3} \partial \alpha_{1}} \frac{\partial A_{1}}{\partial \alpha_{1}}+\frac{\partial w}{A_{1} A_{2}^{2} \partial \alpha_{2}} \frac{\partial A_{1}}{\partial \alpha_{2}}\right) \\
& +\frac{\partial u}{R_{1}^{3} A_{1} \partial \alpha_{1}}+\frac{\partial A_{1}}{A_{2} \partial \alpha_{2}} \frac{v}{R_{1}^{2} R_{2} A_{1}},
\end{aligned}
$$

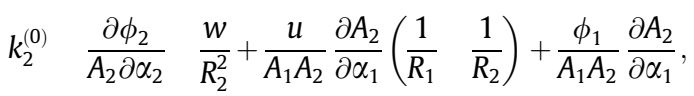

$\left.k_{2}^{(1)} \quad \frac{w}{R_{2}^{3}} \quad \frac{1}{3 R_{2} A_{2}} \quad 2 \frac{\partial \phi_{2}}{\partial \alpha_{2}}+\frac{\partial A_{2}}{A_{2}^{2} \partial \alpha_{2}} \frac{\partial w}{\partial \alpha_{2}} \quad \frac{\partial^{2} w}{A_{2} \partial \alpha_{2}^{2}}\right)$

$$
\left.\frac{\partial A_{2}}{A_{1} A_{2} \partial \alpha_{1}}\left[\begin{array}{ll}
\phi_{1}\left(\frac{1}{R_{2}}\right. & \frac{1}{3 R_{1}}
\end{array}\right)+\frac{u}{R_{2}}\left(\begin{array}{ll}
\frac{1}{R_{1}} & \frac{1}{R_{2}}
\end{array}\right) \quad \frac{\partial w}{3 R_{1} A_{1} \partial \alpha_{1}}\right],
$$

$\left.k_{2}^{(2)} \quad \frac{4}{3 h^{2}} \frac{\partial \phi_{2}}{A_{2} \partial \alpha_{2}}+\frac{\partial^{2} w}{A_{2}^{2} \partial \alpha_{2}^{2}}+\frac{\phi_{1}}{A_{1} A_{2}} \frac{\partial A_{2}}{\partial \alpha_{1}} \quad \frac{\partial w}{A_{2}^{3} \partial \alpha_{2}} \frac{\partial A_{2}}{\partial \alpha_{2}}+\frac{\partial w}{A_{2} A_{1}^{2} \partial \alpha_{1}} \frac{\partial A_{2}}{\partial \alpha_{1}}\right)$ $+\frac{\partial v}{R_{2}^{3} A_{2} \partial \alpha_{2}}+\frac{\partial A_{2}}{A_{1} \partial \alpha_{1}} \frac{u}{R_{2}^{2} R_{1} A_{2}}$

$$
\begin{aligned}
& k_{12}^{(0)} \quad \frac{\partial \phi_{1}}{A_{2} \partial \alpha_{2}}+\frac{\partial \phi_{2}}{A_{1} \partial \alpha_{1}} \quad \frac{\phi_{1}}{A_{1} A_{2}} \frac{\partial A_{1}}{\partial \alpha_{2}} \quad \frac{\phi_{2}}{A_{1} A_{2}} \frac{\partial A_{2}}{\partial \alpha_{1}}
\end{aligned}
$$

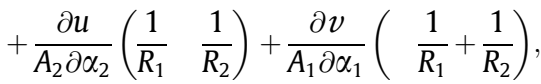

$$
\begin{aligned}
& k_{12}^{(1)} \quad \frac{\partial \phi_{1}}{A_{2} \partial \alpha_{2}}\left(\begin{array}{ll}
\frac{1}{R_{2}} & \frac{1}{3 R_{1}}
\end{array}\right) \quad \frac{\partial \phi_{2}}{A_{1} \partial \alpha_{1}}\left(\begin{array}{ll}
\frac{1}{R_{1}} & \frac{1}{3 R_{2}}
\end{array}\right) \\
& \left.\left.+\frac{\partial u}{A_{2} \partial \alpha_{2}} \quad \frac{1}{R_{2}^{2}} \quad \frac{1}{R_{1} R_{2}}\right)+\frac{\partial v}{A_{1} \partial \alpha_{1}} \quad \frac{1}{R_{1}^{2}} \quad \frac{1}{R_{1} R_{2}}\right) \\
& +\frac{\partial^{2} w}{A_{1} A_{2} \partial \alpha_{1} \partial \alpha_{2}}\left(\frac{1}{3 R_{1}}+\frac{1}{3 R_{2}}\right) \\
& \left.\frac{\partial A_{1}}{A_{2} \partial \alpha_{2}} \quad \frac{2 \partial w}{3 R_{1} A_{1}^{2} \partial \alpha_{1}} \quad \frac{2 \phi_{1}}{3 R_{1} A_{1}}\right) \\
& \left.\frac{\partial A_{2}}{A_{1} \partial \alpha_{1}} \frac{2 \partial w}{3 R_{2} A_{2}^{2} \partial \alpha_{2}} \frac{2 \phi_{2}}{3 R_{2} A_{2}}\right) \text {, }
\end{aligned}
$$

$$
\begin{array}{cc}
k_{12}^{(2)} & \frac{4}{3 h^{2}} \frac{\partial \phi_{1}}{A_{2} \partial \alpha_{2}}+\frac{\partial \phi_{2}}{A_{1} \partial \alpha_{1}}+2 \frac{\partial^{2} w}{A_{1} A_{2} \partial \alpha_{1} \partial \alpha_{2}} 2 \frac{\partial w}{A_{1}^{2} A_{2} \partial \alpha_{1}} \frac{\partial A_{1}}{\partial \alpha_{2}} \\
& \left.2 \frac{\partial w}{A_{1} A_{2}^{2} \partial \alpha_{2}} \frac{\partial A_{2}}{\partial \alpha_{1}} \frac{\phi_{1}}{A_{1} A_{2}} \frac{\partial A_{1}}{\partial \alpha_{2}} \frac{\phi_{2}}{A_{1} A_{2}} \frac{\partial A_{2}}{\partial \alpha_{1}}\right) \\
& \left.\frac{1}{A_{1} A_{2}} \frac{u}{R_{1}^{3}} \frac{\partial A_{1}}{\partial \alpha_{2}}+\frac{v}{R_{2}^{3}} \frac{\partial A_{2}}{\partial \alpha_{1}}\right)+\frac{1}{R_{1} R_{2}}\left(\frac{\partial u}{R_{2} A_{2} \partial \alpha_{2}}+\frac{\partial v}{R_{1} A_{1} \partial \alpha_{1}}\right) .
\end{array}
$$

Eqs. (18d l) give the changes in curvature and torsion of the middle surface, and they have been obtained retaining only linear terms; in fact, nonlinear terms in the changes in curvature and torsion play a very small role, at least for moderate vibration amplitudes [25]. Eqs. (18a c), giving the middle surface strains, 
are coincident with those obtained by using Novozhilov nonlinear shell theory $[1,23]$, which neglects shear deformation and rotary inertia.

\section{Elastic strain and kinetic energies for laminated composite shells}

It is assumed that the transverse normal stress $\sigma_{3} \quad 0$ is negligi ble (plane stress assumption); in general, it is verified that $\sigma_{3}$ is small compared to $\tau_{13}$ and $\tau_{23}$, except near the shell edges, so that the hypothesis is a good approximation of the actual behavior of moderately thick shells. The stress strain relations for the $k$ th orthotropic lamina of the shell, in the material principal coordi nates $(x, y, z)$, are obtained under the hypothesis $\sigma_{3} \quad 0$ [1,7],

$\left\{\begin{array}{l}\sigma_{x} \\ \sigma_{y} \\ \tau_{y z} \\ \tau_{x z} \\ \tau_{x y}\end{array}\right\}^{(k)} \quad\left[\begin{array}{lllll}c_{11} & c_{12} & 0 & 0 & 0 \\ c_{21} & c_{22} & 0 & 0 & 0 \\ 0 & 0 & G_{y z} & 0 & 0 \\ 0 & 0 & 0 & G_{x z} & 0 \\ 0 & 0 & 0 & 0 & G_{x y}\end{array}\right]^{(k)}\left\{\begin{array}{l}\varepsilon_{x x} \\ \varepsilon_{y y} \\ \gamma_{y z} \\ \gamma_{x z} \\ \gamma_{x y}\end{array}\right\}$

where $G_{x y}, G_{x z}$ and $G_{y z}$ are the shear moduli in $x \quad y, x \quad z$ and $y z$ directions, respectively, and the superscript $(k)$ refers to the $k$ th layer within a laminate. The coefficients $c_{i j}$ are given for a lamina by

$$
\begin{aligned}
& c_{11} \frac{E_{x}}{1 v_{x y} v_{y x}}, \quad c_{12} \quad c_{21} \frac{E_{y} v_{x y}}{1 v_{x y} v_{y x}}, \\
& c_{22} \frac{E_{y}}{1 v_{x y} v_{y x}}, \quad v_{i j} E_{j} \quad v_{j i} E_{i} .
\end{aligned}
$$

Eq. (20) is obtained (i) under the transverse isotropy assumption with respect to planes orthogonal to the $x$ axis, i.e. assuming fibers in the direction parallel to axis $x$, so that $E_{y} \quad E_{z}, G_{x y} \quad G_{x z}$ and $v_{x y}=$ $v_{x z}$, and (ii) solving the constitutive equations for $\varepsilon_{z z}$ as function of $\varepsilon_{x x}$ and $\varepsilon_{y y}$ and then eliminating it.

Usually, the lamina material axes $(x, y)$ do not coincide with the shell reference axes $(1,2)$, while the $z$ axis is coincident with 3 . Then, the strains and stresses on material axes can be related to the principal axes by using the following invertible expressions:

$$
\left\{\begin{array}{c}
\sigma_{x} \\
\sigma_{y} \\
\tau_{y z} \\
\tau_{x z} \\
\tau_{x y}
\end{array}\right\} \quad \mathbf{T}_{1}\left\{\begin{array}{c}
\sigma_{1} \\
\sigma_{2} \\
\tau_{23} \\
\tau_{13} \\
\tau_{12}
\end{array}\right\},\left\{\begin{array}{l}
\varepsilon_{x x} \\
\varepsilon_{y y} \\
\gamma_{y z} \\
\gamma_{x z} \\
\gamma_{z y}
\end{array}\right\} \quad \mathbf{T}_{2}\left\{\begin{array}{c}
\varepsilon_{11} \\
\varepsilon_{22} \\
\gamma_{23} \\
\gamma_{13} \\
\gamma_{12}
\end{array}\right\},
$$

where

\begin{tabular}{|c|c|c|c|c|c|}
\hline \multirow{5}{*}{$\mathbf{T}_{2}$} & $\cos ^{2} \beta$ & $\sin ^{2} \beta$ & 0 & 0 & $\sin \beta \cos \beta$ \\
\hline & $\sin ^{2} \alpha$ & $\cos ^{2} \alpha$ & 0 & 0 & $\sin \beta \cos \beta$ \\
\hline & 0 & 0 & $\cos \beta$ & $\sin \beta$ & 0 \\
\hline & 0 & 0 & $\sin \beta$ & $\cos \beta$ & 0 \\
\hline & $2 \sin \beta c c$ & $\sin \beta \cos \beta$ & 0 & 0 & $\cos ^{2} \beta \quad \sin ^{2} \beta$ \\
\hline
\end{tabular}

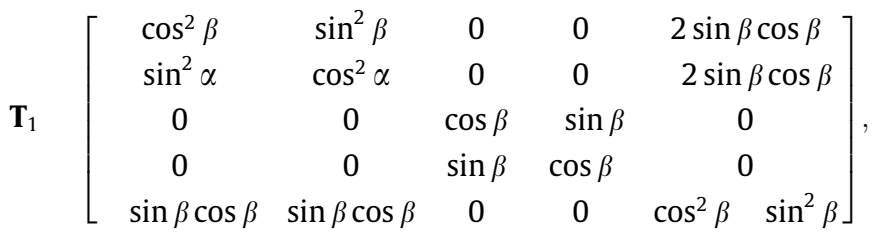

$\beta$ being the angle between the shell principal coordinate $x$ and the material axis 1 as shown in Fig. 3. Also, it can be shown that
$\left(\mathbf{T}_{1}{ }^{1}\right)^{T} \quad \mathbf{T}_{2}$

It is convenient to introduce the matrix $[Q]^{(k)}$ given by

$[Q]^{(k)} \quad\left[\mathbf{T}_{1}{ }^{1} \mathbf{C T}_{2}\right]^{(k)} \quad\left[\mathbf{T}_{2}^{T} \mathbf{C T}_{2}\right]^{(k)}$,

where $\mathbf{C}$ is the $5 \times 5$ matrix of $c_{i j}$ and $G_{i j}$ coefficients in Eq. (19). As a consequence of the discontinuous variation of the stiffness matrix $[Q]^{(k)}$ from layer to layer, the stresses may be discontinuous layer to layer.

Eq. (19) can be transformed to the shell principal coordinates $(1,2,3)$ by the following equation:

$$
\left.\left\{\begin{array}{l}
\sigma_{1} \\
\sigma_{2} \\
\tau_{23} \\
\tau_{13} \\
\tau_{12}
\end{array}\right\} \quad[Q]^{(k)}\left[\begin{array}{l}
\varepsilon_{1,0} \\
\varepsilon_{2,0} \\
\gamma_{23,0} \\
\gamma_{13,0} \\
\gamma_{12,0}
\end{array}\right\}+z\left\{\begin{array}{l}
k_{1}^{(0)} \\
k_{2}^{(0)} \\
k_{23}^{(0)} \\
k_{13}^{(0)} \\
k_{12}^{(0)}
\end{array}\right\}+z^{2}\left\{\begin{array}{l}
k_{1}^{(1)} \\
k_{2}^{(1)} \\
k_{23}^{(1)} \\
k_{13}^{(1)} \\
k_{12}^{(1)}
\end{array}\right\}+z^{3}\left\{\begin{array}{c}
k_{1}^{(2)} \\
k_{2}^{(2)} \\
k_{23}^{(2)} \\
k_{13}^{(2)} \\
k_{12}^{(2)}
\end{array}\right\}\right] .
$$

The elastic strain energy $U_{S}$ of the shell is given by

$$
\begin{aligned}
U_{S} & \frac{1}{2} \sum_{k 1}^{K} \int_{0}^{a} \int_{0}^{b} \\
& \times \int_{h^{(k 1)}}^{h^{(k)}}\left(\sigma_{1}^{(k)} \varepsilon_{11}+\sigma_{2}^{(k)} \varepsilon_{22}+\tau_{12}^{(k)} \gamma_{12}+\tau_{13}^{(k)} \gamma_{13}+\tau_{23}^{(k)} \gamma_{23}\right) \\
& \times\left[1+\left(z / R_{1}\right)\right]\left[1+\left(z / R_{2}\right)\right] A_{1} A_{2} d \alpha_{1} d \alpha_{2} d z,
\end{aligned}
$$

where $K$ is the total number of layers in the laminated shell and $\left(h^{(k 1)}, h^{(k)}\right)$ are the $z$ coordinates of the $k$ th layer, see Fig. 4. For simplicity a shell of rectangular base of curvilinear dimension $a$ and $b$ in $\alpha_{1}$ and $\alpha_{2}$ directions, respectively, has been considered in Eq. (27). Since the stresses are directly related to the strains by Eq. (26) and the strains are a quadratic nonlinear function of the 5 parameters ( 3 displacements of the middle surface and 2 rotations of the normal), the elastic strain energy given by Eq. (27) is an expression with terms up to power four in the partial derivatives of the 5 parameters.

The kinetic energy $T_{S}$ of the shell, including inertia related to all the 5 parameters, is given by

$$
\begin{aligned}
T_{S} & \frac{1}{2} \sum_{k}^{K} \rho_{S}^{(k)} \int_{0}^{a} \int_{0}^{b} \\
& \times \int_{h^{(k 1)}}^{h^{(k)}}\left(\dot{u}_{1}^{2}+\dot{u}_{2}^{2}+\dot{u}_{3}^{2}\right)\left[1+\left(z / R_{1}\right)\right]\left[1+\left(z / R_{2}\right)\right] A_{1} A_{2} d \alpha_{1} d \alpha_{2} d z
\end{aligned}
$$

The $z$ and $z^{3}$ terms vanish after integration on $z$ in the case of a laminate with symmetric density with respect to the $z$ axis. In par ticular, for a laminate with the same density for all the layers and uniform thickness, the following simplified expression is obtained:

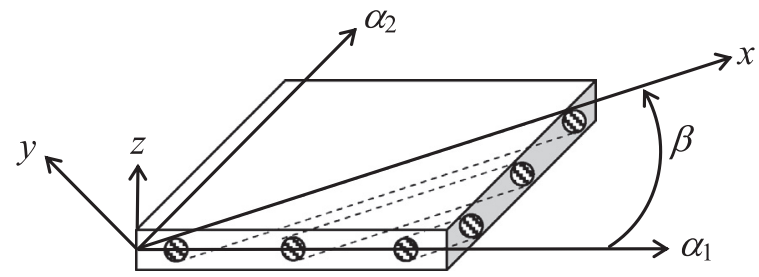

Fig. 3. Shell principal curvilinear coordinates $\left(a_{1}, a_{2}, z\right)$ and material principal coordinates $(x, y, z)$ of the lamina. The fibers inside a lamina and their orientations are shown. 


$$
\begin{aligned}
& T_{S} \frac{1}{2} \rho_{S} h \int_{0}^{a} \int_{0}^{b}\left\{\dot{u}^{2}+\dot{v}^{2}+\dot{w}^{2}+h^{2}\left[\frac{17}{315}\left(\dot{\phi}_{1}^{2}+\dot{\phi}_{2}^{2}\right)\right.\right. \\
& +\dot{\phi}_{1} \dot{u}\left(\frac{29}{90 R_{1}}+\frac{2}{15 R_{2}}\right)+\dot{\phi}_{2} \dot{v}\left(\frac{2}{15 R_{1}}+\frac{29}{90 R_{2}}\right) \\
& +\frac{1}{4}\left(\frac{\dot{u}^{2}}{R_{1}}+\frac{\dot{v}^{2}}{R_{2}}\right)\left(\frac{1}{R_{1}}+\frac{1}{R_{2}}\right)+\frac{\dot{w}^{2}}{12 R_{1} R_{2}}+\frac{\partial \dot{w}}{A_{1} \partial \alpha_{1}} \\
& \times\left(\begin{array}{cccc}
\frac{\partial \dot{w}}{252 A_{1} \partial \alpha_{1}} & \frac{\dot{u}}{90 R_{1}} & \frac{\dot{u}}{30 R_{2}} & \frac{8 \dot{\phi}_{1}}{315}
\end{array}\right)+\frac{\partial \dot{w}}{A_{2} \partial \alpha_{2}} \\
& \left.\left.\times \frac{\partial \dot{w}}{252 A_{2} \partial \alpha_{2}} \quad \frac{\dot{v}}{90 R_{2}} \quad \frac{\dot{v}}{30 R_{1}} \frac{8 \dot{\phi}_{2}}{315}\right)\right\} A_{1} A_{2} d \alpha_{1} d \alpha_{2}+O\left(h^{3}\right) \text {. }
\end{aligned}
$$

\section{Formulation of the theory for circular cylindrical shells}

In order to reduce the system to finite dimensions, the middle surface displacements $u, v$ and $w$, and the two rotations $\phi_{1}$ and $\phi_{2}$ are expanded by using approximate functions. Circular cylindri cal shells with simply supported boundary conditions are analyzed in the following part of the study, as shown in Fig. 5. In particular, $R_{1} \quad \infty, R_{2} \quad R, \alpha_{1} \quad x, \alpha_{2} \quad \theta, A_{1} \quad 1, A_{2} \quad R, a \quad L$ and $b \quad 2 \pi$, which gives the following strain displacement relationships

$$
\begin{aligned}
& \varepsilon_{x, 0} \frac{\partial u}{\partial x}+\frac{1}{2}\left[\left(\frac{\partial u}{\partial x}\right)^{2}+\left(\frac{\partial v}{\partial x}\right)^{2}+\left(\frac{\partial w}{\partial x}\right)^{2}\right]+\frac{\partial w_{0}}{\partial x} \frac{\partial w}{\partial x}, \\
& \varepsilon_{\theta, 0} \quad \frac{1}{R} \frac{\partial v}{\partial \theta}+\frac{w}{R}+\frac{1}{2}\left[\left(\frac{1}{R} \frac{\partial u}{\partial \theta}\right)^{2}+\left(\frac{1}{R} \frac{\partial v}{\partial \theta}+\frac{w}{R}\right)^{2}\right. \\
& \left.+\left(\begin{array}{cc}
\frac{1}{R} \frac{\partial w}{\partial \theta} & \frac{v}{R}
\end{array}\right)^{2}\right]+\frac{w_{0}}{R}\left(\frac{1}{R} \frac{\partial v}{\partial \theta}+\frac{w}{R}\right)+\frac{1}{R} \\
& \times \frac{\partial w_{0}}{\partial \theta}\left(\begin{array}{ll}
\frac{1}{R} \frac{\partial w}{\partial \theta} & \frac{v}{R}
\end{array}\right)
\end{aligned}
$$

$$
\gamma_{x \theta, 0} \quad \frac{\partial v}{\partial x}+\frac{1}{R} \frac{\partial u}{\partial \theta}+\frac{1}{R} \frac{\partial u}{\partial x} \frac{\partial u}{\partial \theta}+\left(\frac{1}{R} \frac{\partial v}{\partial \theta}+\frac{w}{R}\right) \frac{\partial v}{\partial x}
$$$$
+\frac{\partial w}{\partial x}\left(\begin{array}{ll}
\frac{1}{R} \frac{\partial w}{\partial \theta} & \frac{v}{R}
\end{array}\right)+\frac{w_{0}}{R} \frac{\partial v}{\partial x}+\frac{\partial w_{0}}{\partial x}\left(\begin{array}{ll}
\frac{1}{R} \frac{\partial w}{\partial \theta} & \frac{v}{R}
\end{array}\right)+\frac{1}{R}
$$$$
\times \frac{\partial w_{0}}{\partial \theta} \frac{\partial w}{\partial x},
$$

$\gamma_{x z, 0} \quad \phi_{1}+\frac{\partial w}{\partial x}+\frac{\partial u}{\partial x} \phi_{1}+\frac{\partial v}{\partial x}\left(\frac{v}{R}+\phi_{2}\right)$,

$\gamma_{\theta z, 0} \quad \phi_{2}+\frac{1}{R} \frac{\partial w}{\partial \theta}+\left(\frac{w+w_{0}}{R}+\frac{\partial v}{R \partial \theta}\right)\left(\frac{v}{R}+\phi_{2}\right)+\frac{\partial u}{R \partial \theta} \phi_{1}$,

$\boldsymbol{k}_{x}^{(0)} \frac{\partial \phi_{1}}{\partial x}$, $k_{x}^{(1)} \quad 0$,

$$
\left.k_{x}^{(2)} \quad \frac{4}{3 h^{2}} \quad \frac{\partial \phi_{1}}{\partial x}+\frac{\partial^{2} w}{\partial x^{2}}\right)
$$

$k_{\theta}^{(0)} \quad \frac{1}{R} \frac{\partial \phi_{2}}{\partial \theta} \frac{w}{R^{2}}$,

$\left.k_{\theta}^{(1)} \quad \frac{1}{R^{2}} \quad \frac{2}{3} \frac{\partial \phi_{2}}{\partial \theta} \quad \frac{w}{R} \quad \frac{1}{3 R} \frac{\partial^{2} w}{\partial \theta^{2}}\right)$,

$\left.k_{\theta}^{(2)} \quad \frac{4}{3 h^{2}} \frac{1}{R} \frac{\partial \phi_{2}}{\partial \theta}+\frac{1}{R^{2}} \frac{\partial^{2} w}{\partial \theta^{2}}\right)+\frac{1}{R^{4}} \frac{\partial v}{\partial \theta}$,

$k_{x \theta}^{(0)} \quad \frac{1}{R} \frac{\partial \phi_{1}}{\partial \theta}+\frac{\partial \phi_{2}}{\partial x}+\frac{1}{R}\left(\begin{array}{ll}\frac{\partial v}{\partial x} & \frac{1}{R} \frac{\partial u}{\partial \theta}\end{array}\right)$,

$\left.k_{x \theta}^{(1)} \quad \frac{1}{R} \quad \frac{1}{R} \frac{\partial \phi_{1}}{\partial \theta}+\frac{1}{3} \frac{\partial \phi_{2}}{\partial x}+\frac{1}{3 R} \frac{\partial^{2} w}{\partial x \partial \theta}+\frac{1}{R^{2}} \frac{\partial u}{\partial \theta}\right)$,

$k_{x \theta}^{(2)} \quad \frac{4}{3 h^{2}}\left(\frac{1}{R} \frac{\partial \phi_{1}}{\partial \theta}+\frac{\partial \phi_{2}}{\partial x}+\frac{2}{R} \frac{\partial^{2} w}{\partial x \partial \theta}\right)$

$k_{x z}^{(0)} \quad 0$,

$k_{x z}^{(1)} \quad \frac{4}{h^{2}}\left(\phi_{1}+\frac{\partial w}{\partial x}\right)$,

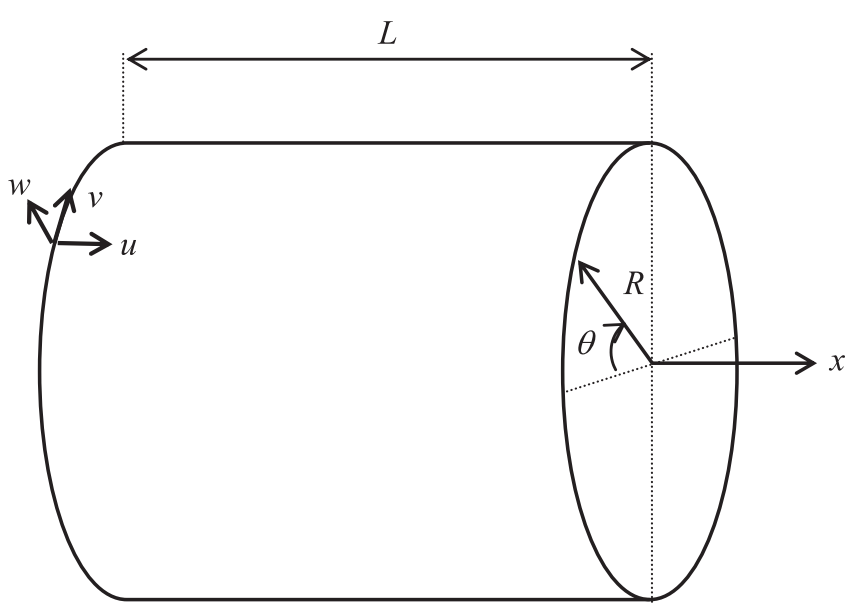

Fig. 5. Coordinate system for a complete circular cylindrical shell.

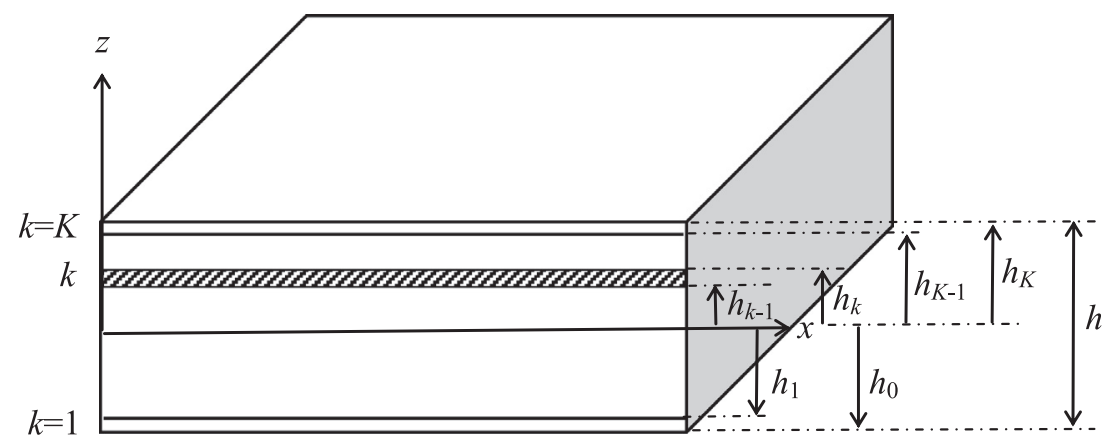

Fig. 4. Notation for thickness of individual layers of a laminate with $K$ layers and global thickness $h$. 
$k_{x z}^{(2)} \quad 0$,

$\boldsymbol{k}_{\theta z}^{(0)} \quad \frac{1}{3 R}\left(\phi_{2}+\frac{\partial w}{R \partial \theta}\right)$,

$k_{\theta z}^{(1)} \quad \frac{4}{h^{2}}\left(\phi_{2}+\frac{1}{R} \frac{\partial w}{\partial \theta}\right)$

$k_{\theta z}^{(2)} \quad \frac{4}{3 h^{2} R}\left(\phi_{2}+\frac{\partial w}{R \partial \theta}\right) \quad \frac{v}{R^{4}}$.

The following boundary conditions are imposed at the shell ends, $x=0, L$ :

$\begin{array}{llllll}w & 0, & v & 0, & \phi_{2} & 0\end{array}$

$N_{x} \quad 0, \quad M_{x} \quad 0$,

where $N_{x}$ is the axial stress resultant per unit length and $M_{x}$ is the axial stress moment resultant per unit length, i.e.,

$$
\left\{\begin{array}{l}
N_{x} \\
M_{x}
\end{array}\right\} \quad \sum_{k 1}^{K} \int_{h^{(k 1)}}^{h^{(k)}} \sigma_{x}^{(k)}\left\{\begin{array}{l}
1 \\
z
\end{array}\right\}[1+(z / R)] d z .
$$

Moreover, the eight parameters must be continuous in $\theta$.

The following base of shell displacements, which satisfy identically the geometric boundary conditions (50a c), is used to discretize the system:

$$
\begin{aligned}
u(x, \theta, t) \quad & \sum_{m}^{M_{1}} \sum_{j 1}^{N}\left[u_{m, j, c}(t) \cos (j \theta)+u_{m, j, s}(t) \sin (j \theta)\right] \cos \left(\lambda_{m} x\right) \\
& +\sum_{m 1}^{M_{2}} u_{m, 0}(t) \cos \left(\lambda_{m} x\right)
\end{aligned}
$$

$v(x, \theta, t) \quad \sum_{m}^{3 M_{1}} \sum_{j 1}^{2 N}\left[v_{m, j, c}(t) \sin (j \theta)+v_{m, j, s}(t) \cos (j \theta)\right] \sin \left(\lambda_{m} x\right)$,

$$
\begin{aligned}
w(x, \theta, t) & \sum_{m}^{M_{1}} \sum_{j}^{N}\left[w_{m, j, c}(t) \cos (j \theta)+w_{m, j, s}(t) \sin (j \theta)\right] \sin \left(\lambda_{m} x\right) \\
& +\sum_{m 1}^{M_{2}} w_{m, 0}(t) \sin \left(\lambda_{m} x\right),
\end{aligned}
$$

$$
\begin{aligned}
\phi_{1}(x, \theta, t) & \sum_{m}^{M_{1}} \sum_{1 j 1}^{N}\left[\phi_{1_{m, j, c}}(t) \cos (j \theta)+\phi_{1_{m j, s}}(t) \sin (j \theta)\right] \cos \left(\lambda_{m} x\right) \\
& +\sum_{m}^{M_{2}} \phi_{1_{m, 0}}(t) \cos \left(\lambda_{m} x\right),
\end{aligned}
$$

$\phi_{2}(x, y, t) \quad \sum_{m}^{M_{1}} \sum_{j}^{N}\left[\phi_{2_{m j, c}}(t) \sin (j \theta)+\phi_{2_{m j, s}}(t) \cos (j \theta)\right] \sin \left(\lambda_{m} x\right)$,

where $j$ is the number of circumferential waves, $m$ is the number of longitudinal half waves, $\lambda_{m} m \pi / L$ and $t$ is the time; $u_{m, j}(t), v_{m, j}(t), w_{m, j}(t), \phi_{1_{m j}}$ and $\phi_{2_{m j}}$ are the generalized coordi nates that are unknown functions of $t$; the additional subscript $c$ or $s$ indicates if the generalized coordinate is associated to cosine or sine function in $\theta$ except for $v$, for which the notation is reversed (no additional subscript is used for axisymmetric terms). The integers $N, M_{1}$ and $M_{2}$ must be selected with care in order to obtain the required accuracy and acceptable dimension of the nonlinear problem. By using a different number of terms in the expansions, it is possible to study the convergence and the accuracy of the solution.

Initial geometric imperfections of the shell are considered only in radial direction. They are assumed to be associated with zero initial stress. The imperfection $w_{0}$ is expanded in the same form of $w$

$$
\begin{aligned}
w_{0}(x, \theta) \quad & \sum_{m}^{\tilde{M}_{1}} \sum_{n=1}^{\tilde{N}}\left[A_{m, n} \cos (n \theta)+B_{m, n} \sin (n \theta)\right] \sin \left(\lambda_{m} x\right) \\
& +\sum_{m}^{\tilde{M}_{2}} A_{m, 0} \sin \left(\lambda_{m} x\right)
\end{aligned}
$$

where $A_{m, n}, B_{m, n}$ and $A_{m, 0}$ are the modal amplitudes of imperfec tions; $N, M_{1}$ and $M_{2}$ are integers indicating the number of terms in the expansion.

An additional term $\hat{u}$ can be added to the expansion of the displacement $u$ in Eqs. (50d e) in order to satisfy exactly the natu ral boundary condition (50d) as shown in details in Appendix A. This term has been used in the numerical calculations in order to increase the accuracy. This term can be useful to have fast convergence of the solution with a smaller number of terms in the expansions.

The expression of the kinetic energy for a laminate with the same density for all the layers and uniform thickness is given by

$$
\begin{aligned}
& T_{S} \frac{1}{2} \rho_{S} h \int_{0}^{2 \pi} \int_{0}^{L}\left\{\dot{u}^{2}+\dot{v}^{2}+\dot{w}^{2}+h^{2}\left[\frac{17}{315}\left(\dot{\phi}_{1}^{2}+\dot{\phi}_{2}^{2}\right)+\frac{2}{15 R} \dot{\phi}_{1} \dot{u}\right.\right. \\
& \left.+\frac{29}{90 R} \dot{\phi}_{2} \dot{v}+\frac{1}{4} \frac{\dot{v}^{2}}{R^{2}}+\frac{\partial \dot{w}}{\partial x} \quad \frac{\partial \dot{w}}{252 \partial x} \quad \frac{\dot{u}}{30 R} \quad \frac{8 \dot{\phi}_{1}}{315}\right) \\
& \left.\left.\left.+\frac{\partial \dot{w}}{R \partial \theta} \quad \frac{\partial \dot{w}}{252 R \partial \theta} \quad \frac{\dot{v}}{90 R} \quad \frac{8 \dot{\phi}_{2}}{315}\right)\right]\right\} R d \theta d x+O\left(h^{3}\right) .
\end{aligned}
$$

\section{Pressure load, harmonic excitation, Lagrange equations and damping}

The pressure load is a specific type of load that depends on the deformation of the shell in Lagrangian description. In fact, pressure is acting in normal direction with respect to the deformed surface, and it is applied to an area that shrinks or expands, according to the shell deformation.

For a generic shell, the virtual work done by pressure $p$, positive in the direction of $w$, is given by $[22,26]$

$W \quad \iint_{\Omega} p\left(u \mathbf{e}_{1}+v \mathbf{e}_{2}+w \mathbf{e}_{n}\right) \cdot \mathbf{e}_{n}^{\prime} A_{1}^{\prime} A_{2}^{\prime} \mathrm{d} \alpha_{1} \mathrm{~d} \alpha_{2}$,

where $\Omega$ is the undeformed surface of the shell, $\mathbf{e}_{1}, \mathbf{e}_{2}, \mathbf{e}_{n}$ are the three unit vectors of the undeformed shell surface, $\mathbf{e}_{n}^{\prime}$ is the normal unit vector to the deformed shell surface, and $A_{1}^{\prime}$ and $A_{2}^{\prime}$ are the Lamé parameters after the shell deformation. For a circular cylindri cal shell, Eq. (55) gives [22,26]

$$
\begin{aligned}
W & \int_{0}^{L} \int_{0}^{2 \pi} p\left\{w\left[\left(1+\frac{\partial u}{\partial x}\right)\left(1+\frac{\partial v}{R \partial \theta}+\frac{w}{R}\right) \quad \frac{\partial v}{\partial x} \frac{\partial u}{R \partial \theta}\right]\right. \\
+ & u\left[\left(1+\frac{\partial v}{R \partial \theta}+\frac{w}{R}\right) \frac{\partial w}{\partial x} \quad \frac{\partial v}{\partial x}\left(\frac{\partial w}{R \partial \theta}+\frac{v}{R}\right)\right] \\
+ & \left.v\left[\left(1+\frac{\partial u}{\partial x}\right)\left(\frac{\partial w}{R \partial \theta}+\frac{v}{R}\right)+\frac{\partial u}{R \partial \theta} \frac{\partial w}{\partial x}\right]\right\} \mathrm{d} x\left(R \pm \frac{h}{2}\right) \mathrm{d} \theta
\end{aligned}
$$

where $(R+h / 2)$ has to be taken for external pressure and $(R \quad h / 2)$ has to be considered for internal pressure. 
Distributed forces per unit area $q_{x}, q_{\theta}$ and $q_{z}$ are applied at the middle surface of the shell in $x, \theta$ and $z$ fixed directions in Lagrangian description, respectively. The virtual work $W$ done by the external non displacement dependent forces (i.e. forces that do not change direction and magnitude during the deformation of the shell) is written as

$W \quad \int_{0}^{2 \pi} \int_{0}^{L}\left(q_{x} u+q_{y} v+q_{z} w\right) d x R d \theta$.

Only loads orthogonal to the shell are considered; therefore, $q_{x} \quad q_{\theta}=0$. The external distributed radial load $q_{z}$ applied to the shell is assumed to be composed by a normal concentrated har monic force of magnitude $f$ applied at a point of coordinates $(x, \theta)$ and positive in $z$ direction

$q_{z} \quad \tilde{f} \delta(x \quad \tilde{x}) \delta(\theta \quad \tilde{\theta}) \cos (\omega t)$,

where $\omega$ is the excitation frequency, $t$ is the time, $\delta$ is the Dirac delta function. In the calculations, the point excitation is assumed to be located at middle length of shell, that is, $x \quad L / 2, \theta \quad 0$. Eq. (57) can be rewritten in the following form:

$W \tilde{f} \cos (\omega t)(w)_{x} \quad L / 2, \theta \quad 0$.

The following notation is introduced for brevity:

$\mathbf{q}\left\{u_{m, n} / h, v_{m, n} / h, w_{m, n} / h, \phi_{1_{m, n}}, \phi_{2_{m, n}}\right\}^{T}$,

$m \quad 1, \ldots, M_{1}$ or $M_{2}$ and $n \quad 0, n, 2 n$.

In Eq. (60) the subscript $c$ or $s$ for the right hand side terms has been dropped for sake of simplicity. A non dimensionalization of the time variables with respect to the shell thickness $h$ has been intro duced in Eq. (60). The generic element of the time dependent vector $\mathbf{q}$ is referred to as $q_{j}$; the dimension of $\mathbf{q}$ is $\bar{N}$, which is the number of degrees of freedom (dofs) used in the mode expansion.

The generalized forces $Q_{j}$ are obtained by differentiation of the virtual work done by external forces:

$Q_{j} \frac{\partial W}{\partial q_{j}}$.

The Lagrange equations of motion are

$\frac{d}{d t}\left(\frac{\partial T_{S}}{\partial \dot{q}_{j}}\right) \quad \frac{\partial T_{S}}{\partial q_{j}}+\frac{\partial U_{S}}{\partial q_{j}} \quad Q_{j}, \quad j \quad 1, \ldots, N$,

where $\partial T_{S} / \partial q_{j} \quad 0$. The complicated term, derived from the maxi mum potential energy of the shell, giving quadratic and cubic non linearities, can be written in the form

$\frac{\partial U_{S}}{\partial q_{j}} \sum_{i=1}^{N} k_{j, i} q_{i}+\sum_{i, k}^{N} s_{j, i, k} q_{i} q_{k}+\sum_{i, k, l}^{N} r_{j, i, k, l} q_{i} q_{k} q_{l}, \quad j \quad 1, \ldots, N$

where the linear coefficients $k_{j, i}$, quadratic coefficients $s_{j, i, k}$ and cubic coefficients $r_{j, i, k, l}$ have long expressions that include also geometric imperfections.

Inertial coupling arises in the equations of motion so that they cannot be immediately transformed in the form required for numerical integration. In particular, Eq. (62) can be written in the following matrix form:

$\mathbf{M} \ddot{\mathbf{q}}+\mathbf{C} \dot{\mathbf{q}}+\left[\mathbf{K}+\mathbf{N}_{2}(\mathbf{q})+\mathbf{N}_{3}(\mathbf{q}, \mathbf{q})\right] \mathbf{q} \quad \mathbf{f}_{\mathbf{0}} \cos (\omega t)+\mathbf{p}_{\mathbf{0}}$,

where $\mathbf{M}$ is the non diagonal mass matrix of dimension $\bar{N} \times \bar{N}$; $\mathbf{C}$ is the viscous damping matrix, which is added to the equations of motion to describe dissipation; $\mathbf{K}$ is the linear stiffness matrix with elements $k_{j, i}, \mathbf{N}_{\mathbf{2}}$ gives the quadratic nonlinear stiffness terms, $\mathbf{N}_{\mathbf{3}}$ denotes the cubic nonlinear terms, $\mathbf{f}_{\mathbf{0}}$ and $\mathbf{p}_{\mathbf{0}}$ are the vectors representing the external harmonic excitation and the static pres sure load, which are obtained by using Eqs. (56), (59) and (61). In this case, $\mathbf{p}_{\mathbf{0}}$ is nonlinear in the middle surface displacements $u, v$ and $w$.

In particular, by using Eq. (63), the generic elements $n_{2_{j, i}}$ and $n_{3_{j, i}}$ of the matrices $\mathbf{N}_{\mathbf{2}}$ and $\mathbf{N}_{\mathbf{3}}$, respectively, are given by

$n_{2_{j, i}}(\mathbf{q}) \quad \sum_{k}^{N} s_{j, i, k} q_{k}, \quad n_{3_{j, i}}(\mathbf{q}, \mathbf{q}) \quad \sum_{k, l 1}^{N} r_{j, i, k, l} q_{k} q_{l}$

In order to obtain the equations of motion in a suitable form for numerical implementation, the system (64) is multiplied by the inverse of mass matrix and then is written in the state space form as follows

$\dot{\mathbf{q}} \mathbf{y}$,

$$
\begin{aligned}
\dot{\mathbf{y}} \quad & \quad \mathbf{M}^{\mathbf{1}} \mathrm{C} \dot{\mathbf{q}}\left[\mathbf{M}{ }^{\mathbf{1}} \mathbf{K}+\mathbf{M}^{\mathbf{1}} \mathbf{N}_{\mathbf{2}}(\mathbf{q})+\mathbf{M}^{\mathbf{1}} \mathbf{N}_{\mathbf{3}}(\mathbf{q}, \mathbf{q})\right] \mathbf{q} \\
+ & \mathbf{M}^{\mathbf{1}} \mathbf{f}_{\mathbf{0}} \cos (\omega t)+\mathbf{M}^{\mathbf{1}} \mathbf{p}_{\mathbf{0}},
\end{aligned}
$$

where $y$ is the vector of the generalized velocities. In particular, the dissipation term is given by

$$
\mathbf{M}^{1} \mathbf{C}\left[\begin{array}{lll}
2 \omega_{1} \zeta_{1} & \ldots & 0 \\
\vdots & \ddots & \vdots \\
0 & \ldots & 2 \omega_{N} \zeta_{N}
\end{array}\right] .
$$

In Eq. (67) $\omega_{i}$ are the natural frequencies and $\zeta_{i}$ the corresponding modal damping ratios of each generalized coordinate. Matrix (67) is assumed to be diagonal in order to use modal damping. The damping identified by experimental modal analysis for small vibra tion amplitude is generally increasing with the vibration amplitude, following a nonlinear relationship. Therefore, in the nonlinear simulations, nonlinear experimental data are important in order to use realistic damping ratios, which are amplitude dependent coefficients.

\section{Numerical methods}

All the variables are made non dimensional to improve numerical accuracy, as shown in Eq. (60). Time is divided by the period of the fundamental natural frequency of the shell in dynamic analysis.

The nonlinear equations of motion have been integrated by using the software AUTO [27] for continuation and bifurcation analysis by using the pseudo arclength continuation method, starting at zero pressure and zero external harmonic force from the trivial solution. The solution has been initially continued with the uniform internal pressure amplitude as parameter up to reach the desired pressure load. Once a pitchfork bifurcation point is reached for external (negative) pressure, which corresponds to buckling, the solution is continued in order to obtain the post buckling configuration.

In case of dynamic analysis, the solution has been continued with the excitation amplitude as parameter and fixed excitation frequency. Once the desired excitation level has been reached, the solution has been continued by using the excitation frequency as continuation parameter. At the pitchfork bifurcation point, a new branch is coming out and corresponds to traveling wave solution around the shell. This traveling wave solution presents Neimark Sacker bifurcations, also detected by using the computer code AUTO, that give rise to quasi periodic vibrations.

\section{Numerical results: nonlinear static analysis}

The dimensions and material properties of a simply supported, imperfection free, laminated circular cylindrical shell made of 

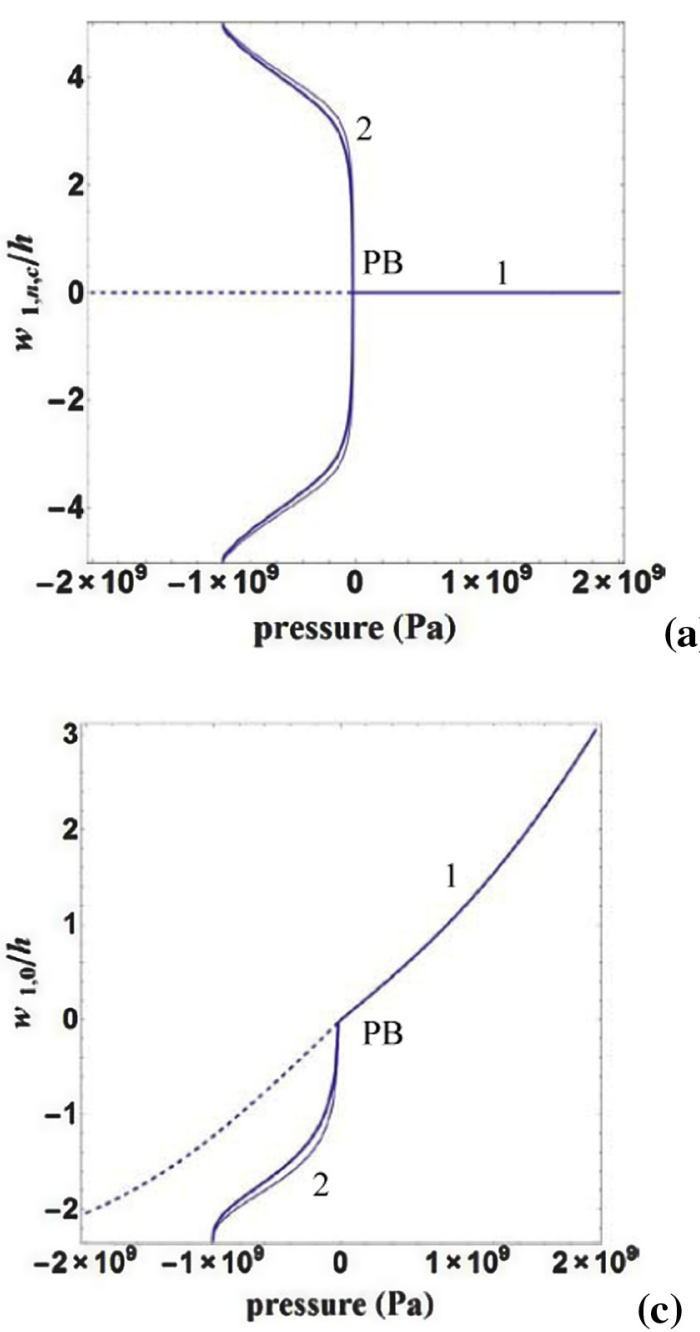

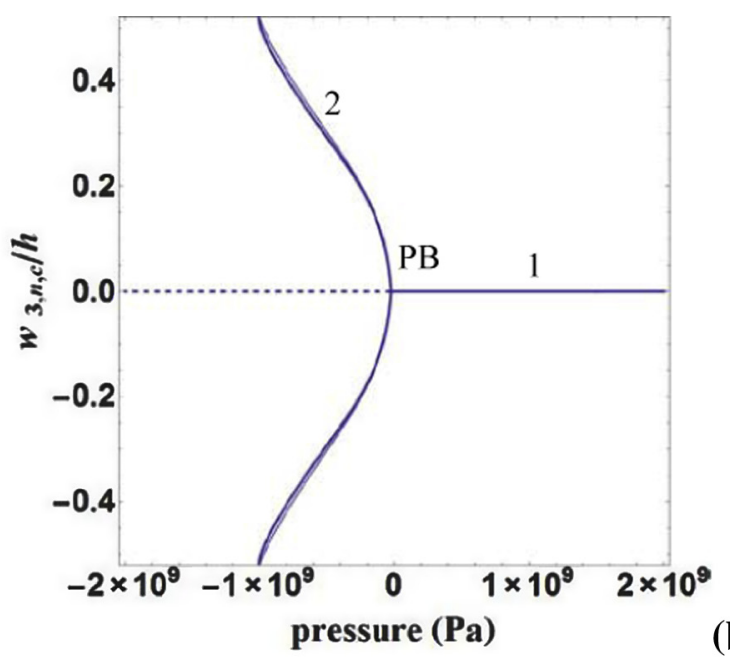

(b)

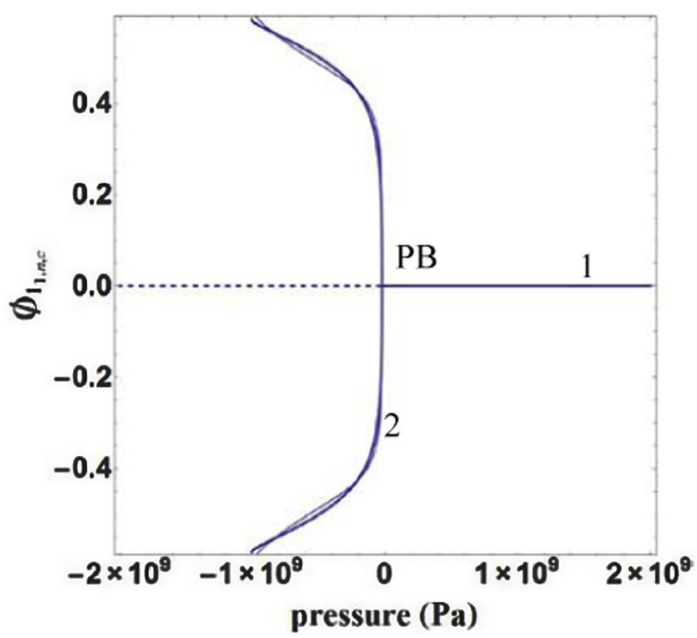

(d)

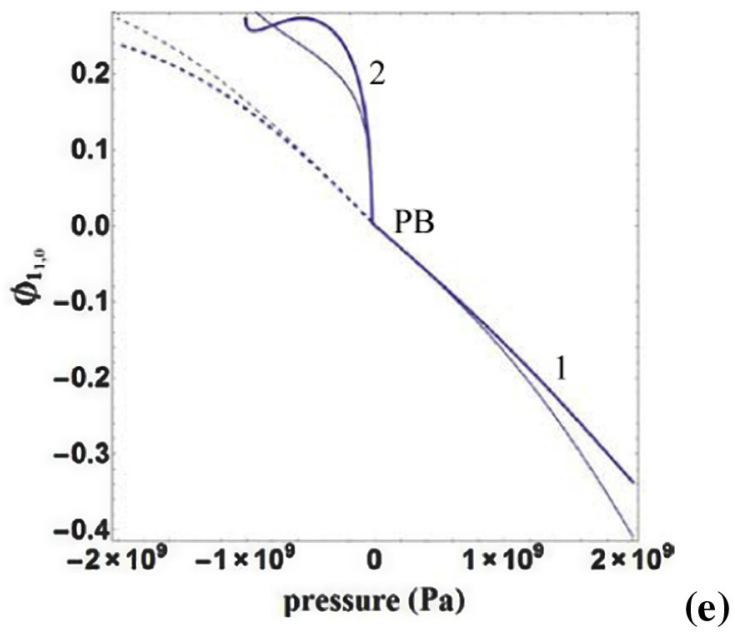

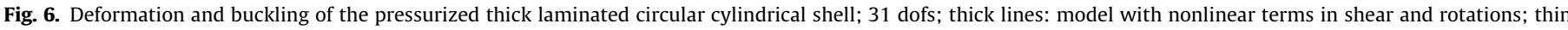

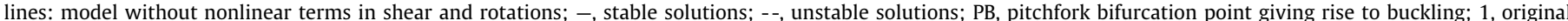

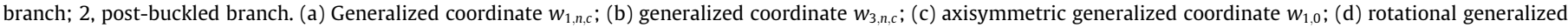
coordinate $\phi_{1, n, c}$; (e) rotational axisymmetric generalized coordinate $\phi_{1,0}$.

graphite/epoxy composite layers are: $R=0.15 \mathrm{~m}, L=0.52 \mathrm{~m}, h=$ $0.03 \mathrm{~m}, E_{1}=50 \times 10_{9} \mathrm{~Pa}, E_{2}=2 \times 10_{9} \mathrm{~Pa}, G_{12} \quad G_{13}=1 \times 10_{9} \mathrm{~Pa}$, $G_{23}=0.4 \times 10_{9} \mathrm{~Pa}, v_{12}=v_{23}=0.25$ and $\rho=1500 \mathrm{~kg} / \mathrm{m}_{3}$. This is a thick shell, being $R / h=5$. The shell is made of four layers $0 / 90^{\circ} /$ $90^{\circ} / 0$ of the same thickness ( $\alpha=0$ for the internal layer, then two layers at $\alpha=\pi / 2$ and finally the external layer with $\alpha=0$ ). The fundamental vibration modes has $n=2$ circumferential waves, so the buckling mode $n=2$ is considered here in the static analysis. In expansion (52a e) the following terms in the summatories are considered different from zero: $w_{1,2}, w_{3,2}, w_{1,0}, w_{3,0}, w_{5,0}, w_{7,0}$, 

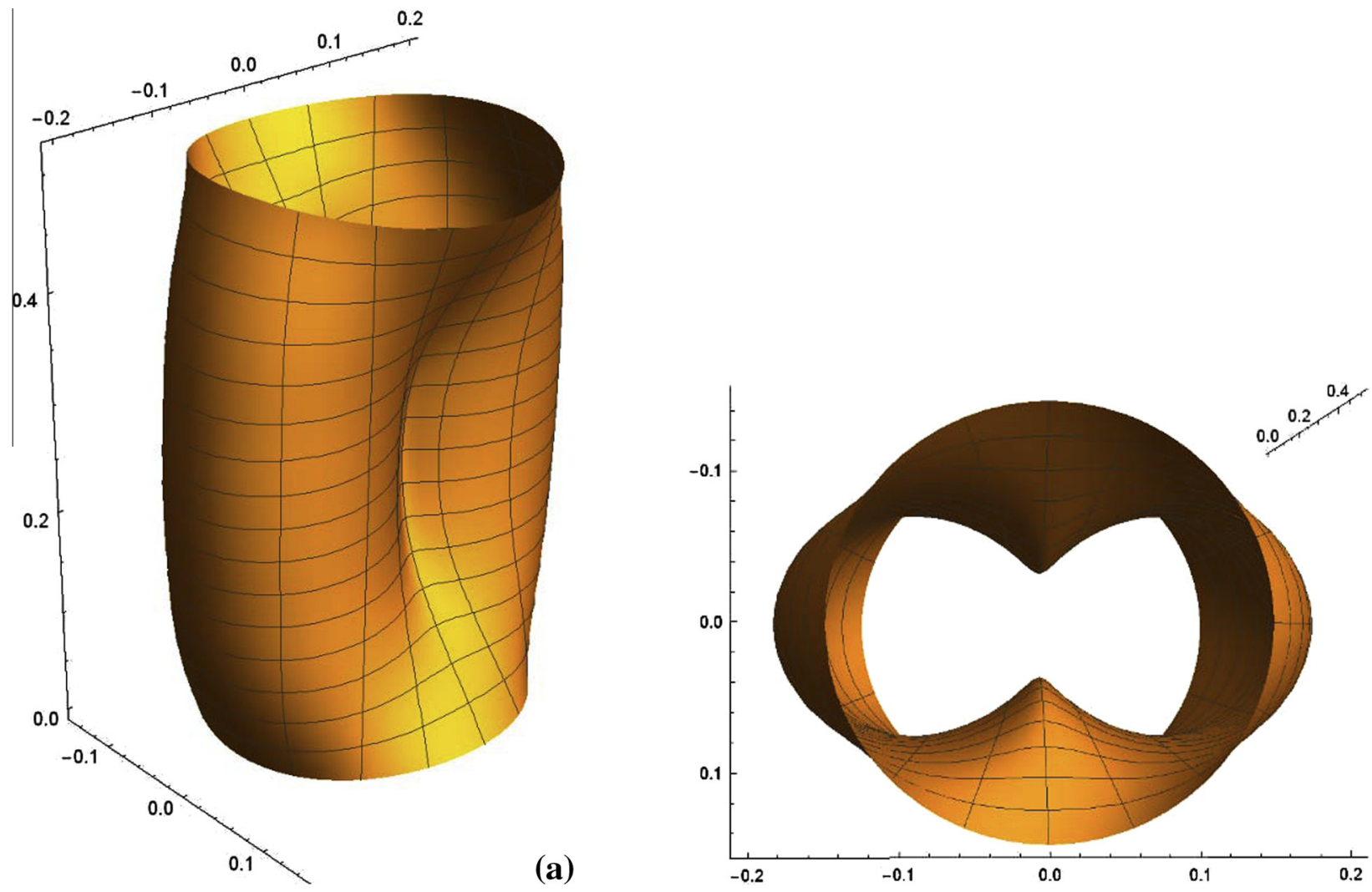

(b)

Fig. 7. Three-dimensional view of the buckled shell for external pressure $-5 \times 10^{7}$ Pa. (a) Viewpoint orthogonal to the $x$ axis; (b) viewpoint along the $x$ axis.

$w_{9,0}, \quad w_{11,0}, u_{1,2}, \quad u_{3,2}, \quad u_{1,0}, \quad u_{3,0}, u_{5,0}, u_{7,0}, u_{9,0}, u_{11,0}, \quad v_{1,2}, v_{3,2}$, $v_{1,4}, \quad v_{3,4}, \quad v_{5,4}, \phi_{1_{1,2}}, \phi_{1_{3,2}}, \phi_{1_{1,0}}, \phi_{1_{3,0}}, \phi_{1_{5,0}}, \phi_{1_{7,0}}, \phi_{1_{9,0}}, \phi_{1_{11,0}}$, $\phi_{2,2}, \phi_{2,2}$; the subscript $c$ has been dropped for brevity in all terms with circumferential wavenumber $n$ different from zero. The expansion used has $\bar{N}=31$ dofs. It allows to study axisymmet ric deformation due to pressure load and postbuckling of mode with 2 circumferential waves $(n=2)$ with accuracy.

The normalized (with respect to the thickness $h$ ) displacements and rotations of the shell versus pressure are presented in Fig. 6. A pitchfork bifurcation appears in Fig. 6(a) (e) for negative pressures. A new branch of the static solution appears after the pitchfork bifurcation and corresponds to the post buckling config uration of the shell. The buckling pressure (i.e. the pressure at which the pitchfork bifurcation appears) is $2.42 \times 10_{7} \mathrm{~Pa}$. While Fig. 6(a) shows the coordinate $w_{1,2}$, corresponding to the buckled shape with $n=2$ circumferential waves and one longitudinal half wave, the coordinate $w_{3,2}$ (with 3 longitudinal half waves) is given in Fig. 6(b) and the axisymmetric radial displacement is presented in Fig. 6(c). The asymmetric rotation $\phi_{1_{1,2}}$ and the axisymmetric rotation $\phi_{1_{10}}$ are shown in Fig. $6(\mathrm{~d})$ and (e), respectively.

Results show that the new branch after the pitchfork bifurca tion is stable; this indicates a supercritical buckling. Both stable (continuous line) and unstable (dashed line) solutions are plotted in Fig. 6(a) (e); in thick line the present solution is given while the solution obtained neglecting the nonlinear terms in rotations and shear strains is shown with a thin line. It is useful to comment that the difference between the two formulations is here quite small for all the generalized coordinates, except for the axisym metric rotation $\phi_{1_{1,0}}$ presented in Fig. 6(e).

The buckled shape of the shell for external pressure $5 \times 10^{7} \mathrm{~Pa}$ is shown in Fig. 7(a) and (b) and clearly shows a large contribution of the mode shape with $n=2$ circumferential waves in addition to an axisymmetric $(n=0)$ shell contraction.

\section{Numerical results: vibrations}

The same circular cylindrical shell studied in Section 7 is inves tigated here. In all the forced vibration simulations, a modal damp ing coefficient $\varsigma_{m, n}=0.001$ has been used for the mode $(m, n)$, for all its generalized coordinates. For the axisymmetric modes $(n=0)$ retained in the expansion (52a e), a damping proportionally increasing with frequency has been assumed. The mode investi gated here is the mode $(1,4)$, i.e. with $n=4$ circumferential waves, having natural frequency of $\omega_{1, n} \quad 1587.45 \mathrm{~Hz}$; this is the third mode of the shell, being modes $(1,2)$ and $(1,3)$ the fundamental and the second mode, respectively. In expansion (52a e) the fol lowing terms in the summatories are considered different from zero: $w_{1,2, c}, w_{1,2, s}, w_{1,0}, w_{3,0}, w_{5,0}, u_{1,2, c}, u_{1,2, s}, u_{1,0}, u_{3,0}, u_{5,0}$,

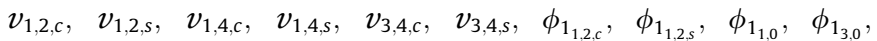
$\phi_{15,0}, \phi_{2,2, c}, \phi_{2,2, s}$. The expansion used has $\bar{N}=23$ dofs and coin cides with the one used in [28]. Here modes with $m=2,4$ axial waves is not considered since the laminate is symmetric and there fore there are no skew modes [29].

Fig. 8(a) (d) present the frequency response curve for forced vibrations of the shell in the frequency neighborhood of the reso nance of the mode $(n=4)$ computed by using the present nonlinear theory (thick continuous and broken lines) and a theory that neglects nonlinear terms in rotations and shear strains (thin continuous and broken lines) that gives results numerically superimposed to those in reference [28] for the same case. A nondimensional harmonic point force excitation of non dimensional amplitude $f=77500 \mathrm{~N}$ and frequency $\omega$ applied at shell mid length is assumed. The branch 1 of the nonlinear 

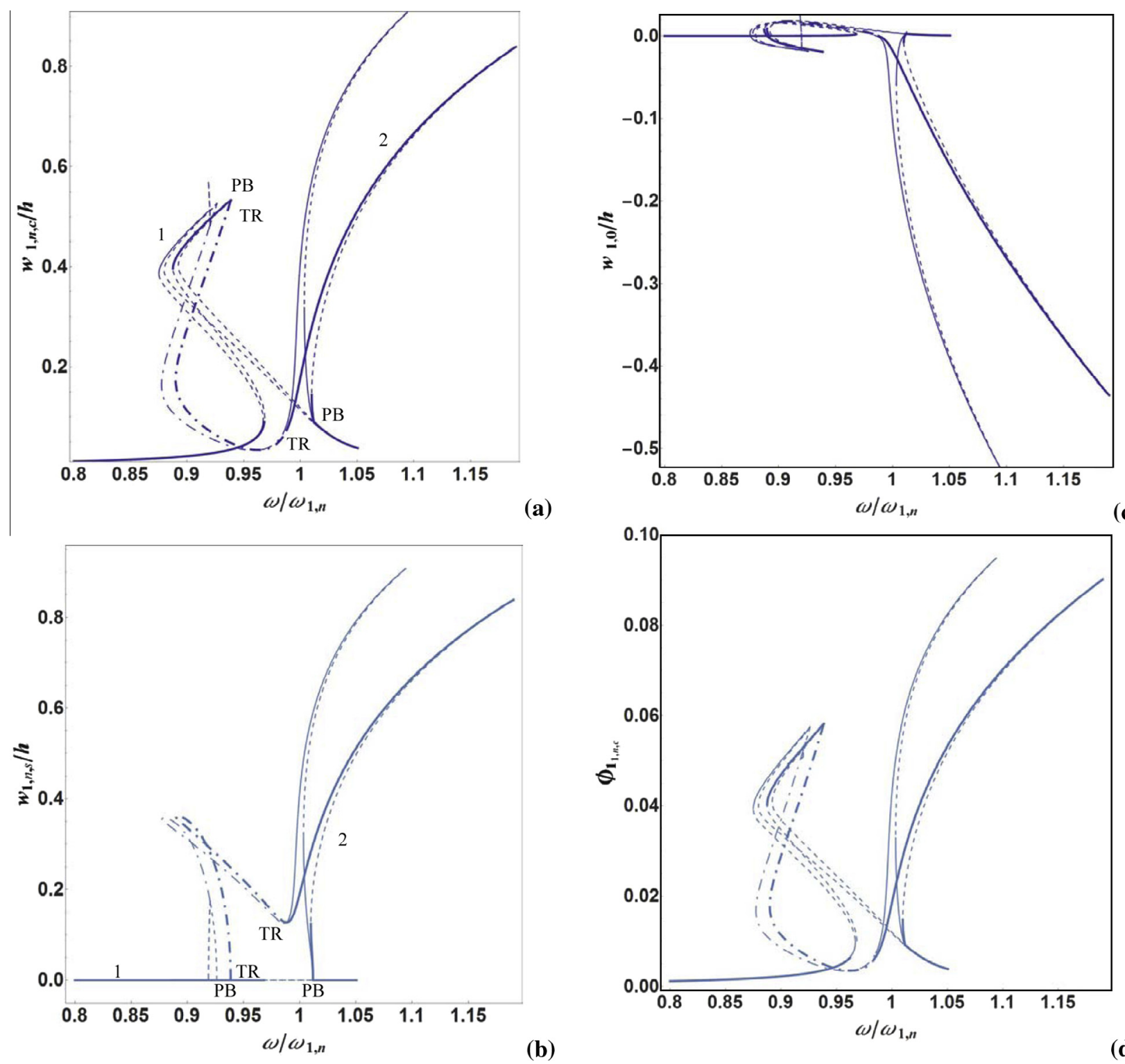

c)

(b)

(d)

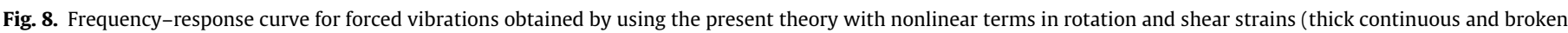

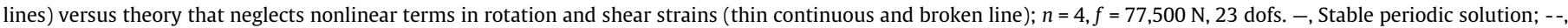

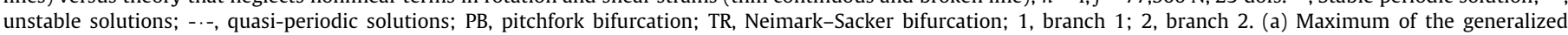

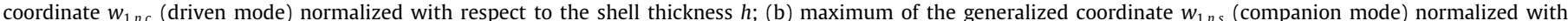

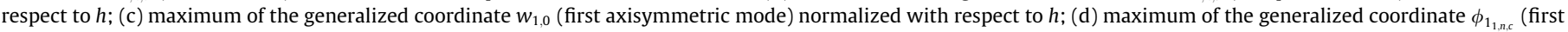
rotation coordinate).

response in Fig. 8 corresponds to vibration with zero amplitude of the companion mode $w_{1, n, S}(t)$. Branch 1 has two pitchfork bifurcations (BP) at $\omega / \omega_{1, n}=0.9385$ and at 1.0118 , where branch 2 appears. This new branch corresponds to participation of both $w_{1, n, c}(t)$ and $w_{1, n, s}(t)$, giving a traveling wave response. The com panion mode presents a node at the location of the excitation force and therefore it is not directly excited; its amplitude is different than zero only for large amplitude vibrations, due to nonlinear coupling through 1:1 internal resonance. In the frequency region where both $w_{1, n, c}(t)$ and $w_{1, n, s}(t)$ are different from zero, they give rise to a traveling wave around the shell; phase shift between the two coordinates is practically equal to $\pi / 2$ when the two generalized coordinates have almost the same amplitude. This branch appears for sufficiently large excitation. Branch 2 under goes two Neimark Sacker (torus) bifurcations (TR), at $\omega / \omega_{1, n}=0.9386$ and 0 . 9.876. Amplitude modulated (quasi periodic) response is indicated in Fig. 3 on branch 2 for $0.9981<\omega / \omega_{1, n}<0.9998$, that is, bracketed by the two Neimark Sacker bifurcations. The maximum of the generalized coordinate $w_{1, n, c}$ (driven mode) normalized with respect to the shell thickness $h$ is shown in Fig. 8(a); the maximum of the generalized coordinate $w_{1, n, s}$ (companion mode) normalized with respect to $h$ is plotted in Fig. 8(b); the maximum of the generalized coordinate $w_{1,0}$ (first axisymmetric mode) normalized with respect to $h$ is presented in Fig. 8(c); and finally Fig. 8(d) gives the maxi mum of the generalized coordinate $\phi_{1_{1, n, c}}$ (first rotation coordinate). The difference between the present theory and the one that neglects nonlinear terms in rotations and shear strains is visible in branch 1 of the solution, but it becomes very significant on branch 2 of the solution corresponding to traveling wave and quasi periodic vibrations.

The time responses of the same generalized coordinates presented in Fig. 8(a) (d) is shown in Fig. 9(a) (e) for excitation frequency $\omega / \omega_{1, n} \quad 1.19$, corresponding to the peak of branch 2 where both 

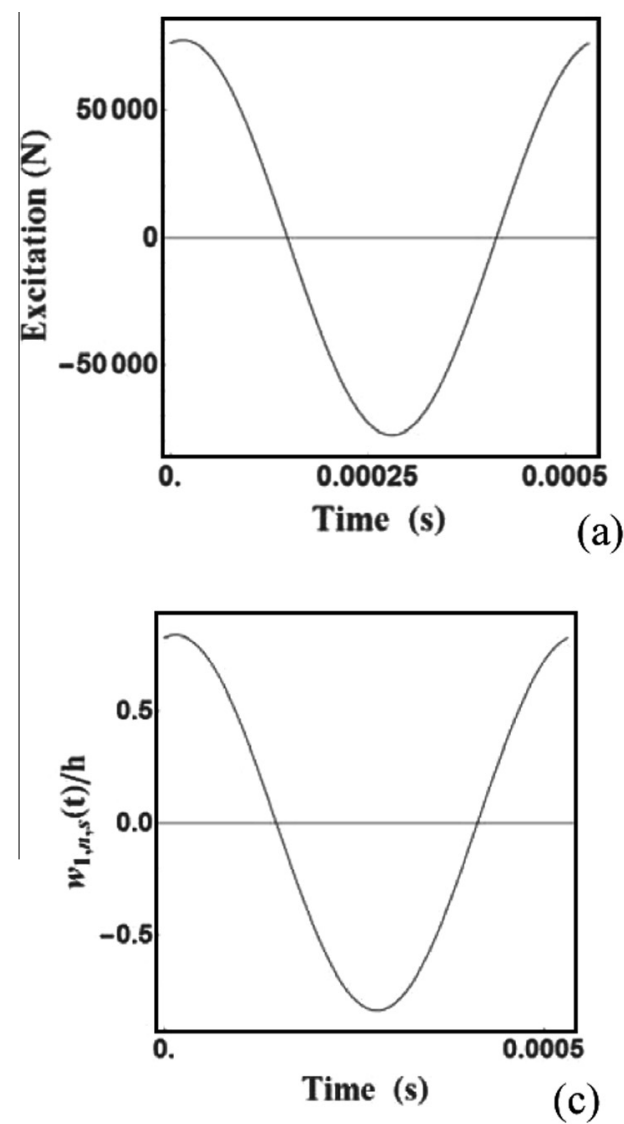
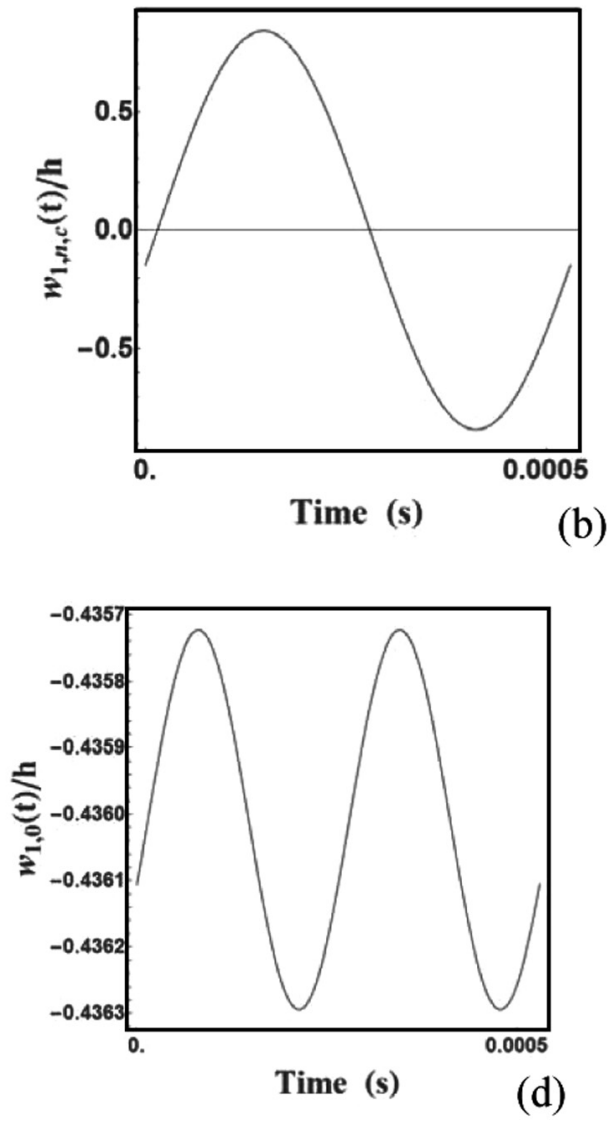

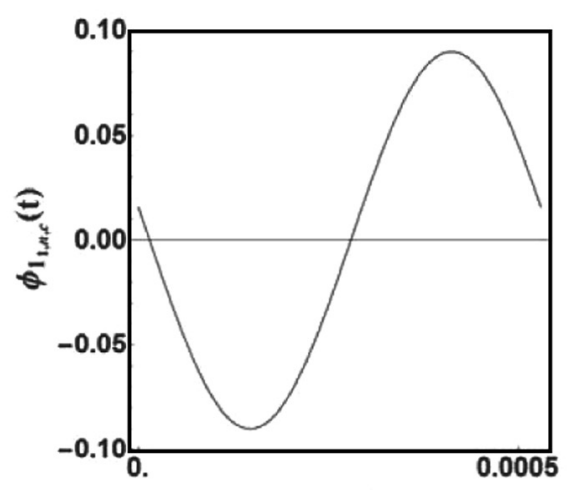

Time (s)

(e)

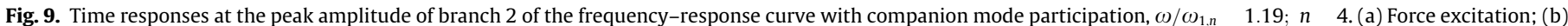

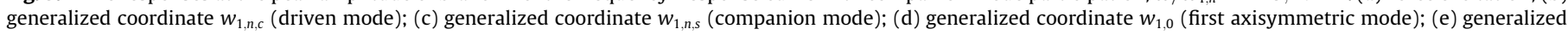
coordinate $\phi_{1, n_{c}}$.

driven and companion modes are active. In particular, Fig. 9(a) shows the force excitation, which is useful to reconstruct phase relation ships. A comparison of Fig. 9(b) and (c) shows that the driven and companion modes have practically the same amplitude but almost a 90 degrees phase difference, giving rise to a traveling wave response around the shell (nodes are traveling around the circumference of the shell). Fig. 9(d) presents the first axisymmetric terms, which gives a contraction to the shell (being always negative) in agreement with the fact that shells are stiffer to in plane stretching with respect to bending. Rotation $\phi_{1, n, c}$ is finally given in Fig. 9(e).

\section{Conclusions}

|The present theory introduces geometrically nonlinear terms in the shear strains for a third order shear deformation theory.
Numerical results show that these terms play a small role in sta tic deformation and buckling in the present case of a pressurized thick cross play laminated composite circular cylindrical shell. However, in case of forced nonlinear vibrations, significant differences are observed for the same shell, indicating that these nonlinear terms should be retained in order to have accurate numerical simulations in dynamics.

\section{Acknowledgments}

The author acknowledges the financial support of the NSERC Discovery Grant and Discovery Accelerator Supplement and Canada Research Chair. 


\section{Appendix A. Additional term to satisfy exactly the boundary conditions}

The natural boundary condition given by Eq. (50d) is not iden tically satisfied by the shell displacements in Eqs. (52a e). In par ticular, after integration with respect to $z$ as shown in Eq. (51), it becomes

$$
\begin{aligned}
& \left.N_{x} \sum_{k 1}^{N}\left\{Q_{11}^{(k)}, Q_{12}^{(k)}, Q_{15}^{(k)}\right\}\left[\left\{\begin{array}{c}
\varepsilon_{x, 0} \\
\varepsilon_{\theta, 0} \\
\gamma_{x \theta, 0}
\end{array}\right\} h^{(k)} \quad h^{(k} \quad 1\right)+\frac{h^{(k) 2}}{2 R}\right) \\
& \left.+\left\{\begin{array}{l}
k_{x}^{(0)} \\
k_{\theta}^{(0)} \\
k_{x \theta}^{(0)}
\end{array}\right\} \frac{h^{(k) 2} h^{(k 1) 2}}{2}+\frac{h^{(k) 3} h^{(k \quad 1) 3}}{3 R}\right)
\end{aligned}
$$

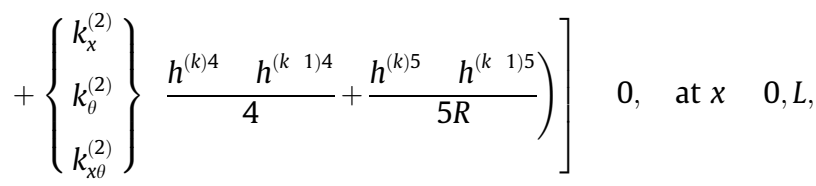

where $Q_{11}^{(k)}, Q_{12}^{(k)}, Q_{15}^{(k)}$ (since $Q_{13}^{(k)} \quad Q_{14}^{(k)} \quad 0$ ) are given by Eq. (25) in Appendix $A$ and the strain displacement relations are given in Eqs. (30) (49). In order to satisfy Eq. (A1) up to second order non linear terms, a second order term $\hat{u}(x, \theta, t)$ must be added to the expansion of $u$ in Eq. (52a). In particular, eliminating in Eq. (A1) null terms at $x=0, L$, the following expression is obtained:

$$
\begin{aligned}
& N_{x}=\sum_{k 1}^{N}\left\{Q_{11}^{(k)}, Q_{12}^{(k)}, Q_{15}^{(k)}\right\}\left[\left\{\begin{array}{c}
\frac{\partial u}{\partial x}+\frac{1}{2}\left[\left(\frac{\partial v}{\partial x}\right)^{2}+\left(\frac{\partial w}{\partial x}\right)^{2}\right]+\frac{\partial w}{\partial x} \frac{\partial w_{0}}{\partial x} \\
\frac{1}{2}\left(\frac{1}{R} \frac{\partial u}{\partial \theta}\right)^{2} \\
\frac{\partial v}{\partial x}+\frac{1}{R} \frac{\partial u}{\partial \theta}
\end{array}\right\}\right. \\
& \left.\left.\times h^{(k)} h^{(k}{ }^{1)}+\frac{h^{(k) 2} h^{(k 1) 2}}{2 R}\right)+\left\{\begin{array}{c}
0 \\
0 \\
k_{x \theta}^{(0)}
\end{array}\right\} \frac{h^{(k) 2} h^{(k 1) 2}}{2}+\frac{h^{(k) 3} h^{(k 1) 3}}{3 R}\right) \\
& \left.\left.+\left\{\begin{array}{l}
0 \\
0 \\
k_{x \theta}^{(2)}
\end{array}\right\} \frac{h^{(k) 4} h^{(k 1) 4}}{4}+\frac{h^{(k) 5} h^{(k 1) 5}}{5 R}\right)\right]=0, \text { at } x=0, L,
\end{aligned}
$$

where the term $\hat{u}(x, \theta, t)$ has been neglected in second order terms. In equation (A2), all the linear terms $\frac{\partial v}{\partial x}+\frac{1}{R} \frac{\partial u}{\partial \theta}, k_{x \theta}^{(0)}$ and $k_{x \theta}^{(2)}$ can be eliminated since they establish a linear relationship which is satis fied by using the minimization of energy in the process of building the Lagrange equations of motion; in fact, this is equivalent to the Rayleigh Ritz method and therefore it is necessary only to satisfy only geometrical boundary conditions. Therefore Eq. (A2) can be simplified into

$$
\begin{array}{r}
N_{x} \quad \sum_{k 1}^{N}\left\{Q_{11}^{(k)}, Q_{12}^{(k)}\right\}\left[\left\{\begin{array}{c}
\left.\frac{\partial \hat{u}}{\partial x}+\frac{1}{2}\left[\left(\frac{\partial v}{\partial x}\right)^{2}+\left(\frac{\partial w}{\partial x}\right)^{2}\right]+\frac{\partial w}{\partial x} \frac{\partial w_{0}}{\partial x}\right\} \\
\frac{1}{2}\left(\frac{1}{R} \frac{\partial u}{\partial \theta}\right)^{2}
\end{array}\right\}\right. \\
\left.\times h^{(k)} h^{(k 1)}+\frac{h^{(k) 2} h^{(k 1) 2}}{2 R}\right) 0, \text { at } x \quad 0, L,
\end{array}
$$

which immediately gives

$$
\begin{aligned}
N_{x} & \left.=\sum_{k 1}^{N} h^{(k)} h^{(k 1)}+\frac{h^{(k) 2} h^{(k 1) 2}}{2 R}\right)\left[Q_{11}^{(k)} \frac{\partial \hat{u}}{\partial x}+\frac{1}{2}\left[\left(\frac{\partial v}{\partial x}\right)^{2}+\left(\frac{\partial w}{\partial x}\right)^{2}\right]\right. \\
& \left.\left.+\frac{\partial w}{\partial x} \frac{\partial w_{0}}{\partial x}\right)+Q_{12}^{(k)} \frac{1}{2}\left(\frac{1}{R} \frac{\partial u}{\partial \theta}\right)^{2}\right]=0, \text { at } x=0, L .
\end{aligned}
$$

By introducing the notation

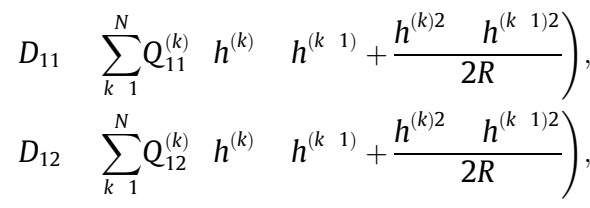

Eq. (A4) can be expressed in the following form

$$
\begin{gathered}
N_{x} \quad D_{11}\left(\frac{\partial \hat{u}}{\partial x}+\frac{1}{2}\left[\left(\frac{\partial v}{\partial x}\right)^{2}+\left(\frac{\partial w}{\partial x}\right)^{2}\right]+\frac{\partial w}{\partial x} \frac{\partial w_{0}}{\partial x}\right) \\
+D_{12} \frac{1}{2}\left(\frac{1}{R} \frac{\partial u}{\partial \theta}\right)^{2} \quad 0, \text { at } x \quad 0, L .
\end{gathered}
$$

In Eq. (A6) it is necessary to retain only the resonant mode $(m, n)$ and, in case of pressurization, the axisymmetric modes. The expression of $\hat{u}$ can be obtained from Eq. (A6) as

$$
\begin{aligned}
\hat{u}(t) \quad & \frac{1}{8}[a(t)+b(t) \cos (2 n \theta)+c(t) \sin (2 n \theta)] \sin (2 m \pi x / L) \\
& \frac{m \pi}{L}\left[w_{m, n, c}(t) \cos (n \theta)+w_{m, n, s}(t) \sin (n \theta)\right] \\
& \left\{\sum_{i=1}^{M_{2}} \frac{i}{m+i} w_{i, 0}(t) \sin [(m+i) \pi x / L] \sum_{j}^{N} \sum_{i}^{\tilde{M}_{1}} \frac{i}{m+i}\right. \\
& {\left.\left[A_{i, j} \cos (j \theta)+B_{i, j} \sin (j \theta)\right] \sin [(m+i) \pi x / L]\right\} } \\
& \frac{1}{2} \sum_{m}^{M_{2}} \frac{m \pi}{L} w_{m, 0}(t) \sum_{i=1}^{M_{2}} \frac{i}{m+i} w_{i, 0}(t) \sin [(m+i) \pi x / L],
\end{aligned}
$$

where

$$
\begin{aligned}
& a(t) \quad(m \pi / L)\left(w_{m, n, c}^{2}+w_{m, n, s}^{2}+v_{m, n, c}^{2}+v_{m, n, s}^{2}\right) \\
& +\left(D_{12} / D_{11}\right)\left[L n^{2} /\left(m \pi R^{2}\right)\right]\left(u_{m, n, c}^{2}+u_{m, n, s}^{2}\right), \\
& b(t) \quad(m \pi / L)\left(\begin{array}{lll}
w_{m, n, c}^{2} & w_{m, n, s}^{2}+v_{m, n, s}^{2} & v_{m, n, c}^{2}
\end{array}\right) \\
& +\left(D_{12} / D_{11}\right)\left[L n^{2} /\left(m \pi R^{2}\right)\right]\left(\begin{array}{ll}
u_{m, n, s}^{2} & u_{m, n, c}^{2}
\end{array}\right), \\
& c(t) \quad(2 m \pi / L)\left(w_{m, n, c} w_{m, n, s}+v_{m, n, c} v_{m, n, s}\right) \\
& 2\left(D_{12} / D_{11}\right)\left[\operatorname{Ln}^{2} /\left(m \pi R^{2}\right)\right] u_{m, n, c} u_{m, n, s} .
\end{aligned}
$$

The boundary condition (50e) does not introduce any additional nonlinear term for symmetric laminates if the term $z / R$ is neglected in Eq. (51), i.e. for thin shells. Additional terms must be added to the expansion of the in plane displacement $u$ for asymmetric laminates and moderately thick shells. In fact, bending and stretching are coupled for asymmetric laminates.

\section{References}

[1] Amabili M. Nonlinear vibrations and stability of shells and plates. NY, USA: Cambridge University Press; 2008.

[2] Amabili M, Farhadi S. Shear deformable versus classical theories for nonlinear vibrations of rectangular isotropic and laminated composite plates. J Sound Vib 2009;320:649-67.

[3] Reddy JN. Mechanics of laminated composite plates and shells: theory and analysis. 2nd ed. Boca Raton, FL, USA: CRC Press; 2004.

[4] Reddy JN. Exact solutions of moderately thick laminated shells. J Eng Mech 1984;110:794-809.

[5] Dennis ST, Palazotto AN. Large displacement and rotation formulation for laminated shells including parabolic transverse shear. Int J Non-Linear Mech 1990;25:67-85.

[6] Palazotto AN, Dennis ST. Nonlinear analysis of shell structures. Washington, DC, USA: AIAA educational series. The American Institute of Aeronautics and Astronautics; 1992.

[7] Amabili M, Reddy JN. A new non-linear higher-order shear deformation theory for large-amplitude vibrations of laminated doubly curved shells. Int J Nonlinear Mech 2010;45:409-18.

[8] Carrera E. A study of transverse normal stress effects on vibration of multilayered plates and shells. J Sound Vib 1999;225:803-29. 
[9] Alijani F, Amabili M. Non-linear vibrations of shells: a literature review from 2003 to 2013. Int J Non-linear Mech 2014;58:233-57.

[10] Carrera E. Transverse normal stress effects in multilayered plates. J Appl Mech 1999;66:1004-12.

[11] Carrera E, Brischetto S, Cinefra M, Soave M. Effects of thickness stretching in functionally graded plates and shells. Compos: Part B 2011;42:123-33.

[12] Ferreira AJM, Carrera E, Cinefra M, Roque CMC. Analysis of laminated doublycurved shells by a layerwise theory and radial basis functions collocation, accounting for through-the-thickness deformations. Comput Mech 2011;48:13-25.

[13] Parisch H. A continuum-based shell theory for non-linear applications. Int J Numer Methods Eng 1995;38:1855-83.

[14] Sansour C. A theory and finite element formulation of shells at finite deformations involving thickness change: circumventing the use of a rotation tensor. Arch Appl Mech 1995;65:194-216.

[15] Sansour C, Wagner W, Wriggers P, Sansour J. An energy-momentum integration scheme and enhanced strain finite element for the non-linear dynamics of shells. Int J Non-linear Mech 2002;37:951-66.

[16] Arciniega RA, Reddy JN. Tensor-based finite element formulation for geometrically nonlinear analysis of shell structures. Comput Methods Appl Mech Eng 2007; 196:1048-73.

[17] Arciniega RA, Reddy JN. Large deformation analysis of functionally graded shells. Int J Solids Struct 2007;44:2036-52.

[18] Payette GS, Reddy JN. A seven-parameter spectral/hp finite element formulation for isotropic, laminated composite and functionally graded shell structures. Comput Methods Appl Mech Eng 2014;278:664-704.

[19] Amabili M. A new nonlinear higher-order shear deformation theory with thickness variation for large-amplitude vibrations of laminated doubly curved shells. J Sound Vib 2013;332:4620-40.
[20] Amabili M. A non-linear higher-order thickness stretching and shear deformation theory for large-amplitude vibrations of laminated doubly curved shells. Int J Non-linear Mech 2014;58:57-75.

[21] Alijani F, Amabili M. Non-linear static bending and forced vibrations of rectangular plates retaining non-linearities in rotations and thickness deformation. Int J Non-linear Mech 2014;67:394-404.

[22] Amabili M. Non-linearities in rotation and thickness deformation in a new third-order thickness deformation theory for static and dynamic analysis of isotropic and laminated doubly curved shells. Int J Non-linear Mech 2015;69:109-28.

[23] Novozhilov VV. Foundations of the nonlinear theory of elasticity. Rochester, NY, USA: Graylock Press; 1953. now available from Dover, NY, USA

[24] Novozhilov VV. Thin shell theory. 2nd ed. Groningen, The Netherlands: Noordhoff; 1964.

[25] Amabili M. Comparison of shell theories for large-amplitude vibrations of circular cylindrical shells: Lagrangian approach. J Sound Vib 2003;264: 1091-125.

[26] Amabili M, Breslavsky ID. Displacement dependent pressure load for finite deflection of shells and plates. Int J Non-linear Mech; submitted for publication.

[27] Doedel EJ, Champneys AR, Fairgrieve TF, Kuznetsov YA, Sandstede B, Wang X. AUTO 97: continuation and bifurcation software for ordinary differential equations (with HomCont), Concordia University, Montreal, Canada; 1998.

[28] Amabili M. Nonlinear vibrations of laminated circular cylindrical shells: comparison of different shell theories. Compos Struct 2011;94:207-20.

[29] Amabili M. Nonlinear vibrations of angle-ply laminated circular cylindrical shells: skewed modes. Compos Struct 2012;94:3697-709. 